\title{
Network aggregation improves gene function prediction of grapevine gene co-expression networks
}

\author{
Darren C. J. Wong ${ }^{1}$
}

Received: 31 May 2019 / Accepted: 21 March 2020

(c) Springer Nature B.V. 2020

\begin{abstract}
Key message Aggregation across multiple networks highlights robust co-expression interactions and improves the functional connectivity of grapevine gene co-expression networks.

Abstract In recent years, the rapid accumulation of transcriptome datasets from diverse experimental conditions has enabled the widespread use of gene co-expression network (GCN) analysis in plants. In grapevine, GCN analysis has shown great promise for gene function prediction, however, measurable progress is currently lacking. Using accumulated microarray datasets from the grapevine whole-genome array (33 experiments, 1359 samples), we explored how meta-analysis through aggregation influences the functional connectivity (performance) of derived networks using guilt-by-association neighbor voting. Two annotation schemes, i.e. MapMan BIN and Pfam, at two sparsity thresholds, i.e. top 100 (stringent) and 300 (relaxed) ranked genes were evaluated. We observed that aggregating across multiple networks improves performance dramatically, with the aggregate outperforming the majority of functional terms across individual networks. Network sparsity and size (i.e. the number of samples and aggregates) were key factors influencing performance while the choice of annotation scheme had little. Systematic comparison with various state-of-the-art microarray and RNA-seq networks was also performed, however, none outperformed the aggregate microarray network despite having good predictive performance. Repeating these series of tests using a functional enrichment-based performance metric also showed remarkably consistent findings with guilt-by-association neighbor voting. To demonstrate its functionality, we explore the function and transcriptional regulation of grapevine EXPANSIN genes. We envisage that network aggregation will offer new and unique opportunities for gene function prediction in future grapevine functional genomics studies. To this end, we make the aggregate networks and associated metadata publicly available at VTC-Agg (https://sites.google.com/view/vtc-agg).
\end{abstract}

Keywords Vitis vinifera $\cdot$ Network aggregation $\cdot$ Co-expression $\cdot$ Meta-analysis $\cdot$ Transcriptome $\cdot$ EXPANSIN

\section{Introduction}

Fundamental to the construction and analysis of gene coexpression networks (GCN) is the 'guilt-by-association' (GBA) principle (Gillis and Pavlidis 2012). This principle is based on the notion that genes that share common functions are often coordinately regulated across multiple

Electronic supplementary material The online version of this article (https://doi.org/10.1007/s11103-020-01001-2) contains supplementary material, which is available to authorized users.

Darren C. J. Wong

wongdcj@gmail.com

1 Ecology and Evolution, Research School of Biology, The Australian National University, Acton, ACT 2601, Australia experiments (Verleyen et al. 2015). Conventionally, GCNs are constructed from correlations (or related similarity measures) derived from the analysis of several experimental projects as a whole, in which tens to the low hundreds of samples often constitute each project. Such an approach is now a commonplace in numerous plant GCNs (Ruprecht et al. 2017; Obayashi et al. 2018; Proost and Mutwil 2018) and has allowed gene-function predictions to be made with high statistical confidence or more recently, allow the evolution of biological pathways across multiple species to be studied, among others (Ruprecht et al. 2017). For these reasons, GCN analysis has gained recent attention in grapevine functional genomics research (reviewed in Wong and Matus 2017). In grapes, gene co-expression network analyses have been used to ascribe potential gene functions to specific genes (Loyola et al. 2016; Malacarne et al. 2016; Amato et al. 2017; Sun 
et al. 2018) and gene families (Grimplet et al. 2016; Moretto et al. 2016; Wong et al. 2016, 2018) and to understand the regulation of various metabolic pathways (Wong et al. 2013; Malacarne et al. 2018). Other studies have focussed on GCNs within a 'condition-dependent' context such as stress responses (Dal Santo et al. 2016; Savoi et al. 2016; Savoi et al. 2017) and fruit development/ripening (Palumbo et al. 2014; Massonnet et al. 2017) or the identification of cisregulatory element (CRE)-driven GCNs (Wong et al. 2017).

In grapevine, the bulk majority of GCN analysis in the last decade have been performed with the Nimblegen whole-genome array datasets, however, recent endeavors to construct RNA-seq GCNs have also been reported (Ohyanagi et al. 2015; Wong et al. 2017; Obayashi et al. 2018; Proost and Mutwil 2018; Vannozzi et al. 2018). In almost all known recent reports, conventional (non-aggregated) means of GCN construction has been performed. The latter involves summarizing all datasets as a whole and applying some form of co-expression metric. The rank of correlation measure (e.g. PCC rank, MR, and HRR) (Obayashi and Kinoshita 2009; Ruprecht et al. 2017; Obayashi et al. 2018; Proost and Mutwil 2018) is one such robust and widelyadopted metric in many recent plant GCN including grapevines. Causal GCNs based on the gaussian graphical model (GGM) have also been constructed using the VESPUCCI expression compendium (Malacarne et al. 2018). Compared to GCN resources for model plant systems that are plentiful, there are only a few publicly-available databases for GCN analysis in grapevine. Resources such as VTCdb (Wong et al. 2013), VESPUCCI (Moretto et al. 2016), ATTED-II (Obayashi et al. 2018), and CoNekT (Proost and Mutwil 2018) provide excellent resources for customized genefunction interrogation.

In non-plant systems where transcriptome datasets are plentiful, cumulative evidence have indicated that meta-analysis approaches-by means of aggregating individual GCNs from multiple network inference methods (Verleyen et al. 2015) or individual experiments/projects (Gillis and Pavlidis 2011; Ballouz et al. 2015; Liesecke et al. 2019)—can improve the recovery of biologically-relevant associations. The underlying rank-and-summate aggregation strategy is simple, yet effective in producing a robust network of high biological relevance by prioritizing recurrent interactions across individual networks or inference methods. Furthermore, in the case where new datasets become available, individual GCN can be constructed and seamlessly integrated into the aggregate. The area under the receiver operator characteristic curve (AUROC) is a popular metric to evaluate the functional connectivity (performance) of GCNs (Ballouz et al. 2017). It assesses the degree to which association within a given gene set can be predicted by an input set using a given annotation scheme such as Gene Ontology (GO), Protein families (Pfam), and MapMan BIN. Therefore, when genes within a given gene set are more likely to have one another as neighbors, a high AUROC value, and thus better-performing network generally applies: i.e. in most network settings, AUROC of 0.5, 0.7, and 0.9 are well-accepted proxies for random, good, and high function performance, respectively (Ballouz et al. 2017).

In plants, the role of network aggregation on the functional connectivity of GCNs has not been thoroughly investigated until recently (Huang et al. 2017; Liesecke et al. 2019). In one comprehensive study, Liesecke et al. (2019) leveraged several large datasets ranging between 600 and 10,000 samples from Arabidopsis, tomato, and maize and found a moderate positive correlation between aggregate size (i.e. the number of aggregates) and GO AUROC performance. Nonetheless, they showed that a few networks obtained from full (or randomly sampled subsets) datasets of reasonably large size (i.e. $>100$ samples) showed comparable performance with the aggregate, suggesting that the former are already sufficiently robust and do not necessarily benefit from network aggregation. As exemplified in one study using human, rice, and yeast datasets, GCNs constructed from large sample sizes (ca. 1000-2000) or a subset of these (ca. 300-500) often suffice to infer a large number of robust associations (Gibson et al. 2013). Furthermore, in model species such as Arabidopsis, humans, mouse, and rat where transcriptomic datasets are plentiful, a saturation of performance was often evident with GCNs constructed from very large datasets (e.g. $>10,000$ samples) (Aoki et al. 2016; Obayashi et al. 2019). Therefore, the prospect of utilizing network aggregation may be limited in these circumstances or deserve deeper investigation. Indeed, Liesecke et al. (2019) showed that the use of different platforms (microarray and RNA-seq), partitioning strategies (random-, $k$-means-, and project-partitioned), and aggregation methods (co-occurrence, HRR-based) are among the key factors that can influence the performance of individual and aggregate plant GCNs. Consequently, several recommendations were put forth based on their extensive evaluation, however, careful optimization was still required, as controlling for factors that maximize performance in one plant system and expression platform combination (Arabidopsis RNA-seq dataset) may not generally apply to others (i.e. tomato and maize microarray dataset) (Liesecke et al. 2019).

By taking advantage of a large compendium of microarray datasets, we explore how meta-analysis through network aggregation influences the functional connectivity (performance) of grapevine GCNs. A systematic comparison of the aggregate against individual and several state-of-the-art microarray- and RNA-seq-derived GCNs were also conducted. This study will prioritise the MapMan BIN annotation and GBA-based neighbor voting for performance evaluation across two sparsity thresholds suited for designing functional studies (Obayashi et al. 2018): i.e. top 100 and 
300 co-expressed genes. Finally, examples of how the aggregate networks can be used to confirm established functions and identify novel associations will be presented.

\section{Materials and methods}

\section{Compilation of microarray data sets and functional gene annotation}

Thirty-three separate experiments encompassing 1359 microarray data sets performed on the $29 \mathrm{~K}$ NimbleGen Grape Whole-genome platform capable of profiling the expression of 28,811 genes were downloaded from Gene Expression Omnibus (Barrett et al. 2013) and Arrayexpress (Rustici et al. 2013). Raw intensity data were summarized for each experimental project independently and as a whole with oligo $\mathrm{R}$ package using the robust multiarray average method (Carvalho and Irizarry 2010). The pre-annotated protein family (Pfam) functional category of the 12Xv1 genome prediction was downloaded from CRIBI Grape Genome Database (Vitulo et al. 2014). Additional functional annotation of genes using the plant-specific MapMan BIN category (Thimm et al. 2004) was performed using Mercator with default settings (Lohse et al. 2014).

\section{Individual (non-aggregate) and aggregated gene co-expression network construction}

For each expression dataset (i.e. individual GCN), a PCC rank (PR) network was constructed (Obayashi and Kinoshita 2009). Briefly, the Pearson's correlation coefficient (PCC) of each gene against all other genes was first calculated and ranked according to descending PCC. The top 100 (stringent) and 300 (relaxed) ranked genes, roughly equating to a per-gene sparsity/threshold of $0.34 \%$ and $1.04 \%$ of the 28,811 represented genes, were evaluated. For the aggregated GCN, the frequency of co-expression interaction(s) present across individual GCNs was used as edge weights and ranked in descending order. As for the case of individual GCNs, per-gene sparsity of $0.34 \%$ and $1.04 \%$, regardless of a minimum number of co-expression interactions, were also used for constructing the final aggregate GCN. For comparative purposes with non-aggregated microarray networks, the PR, mutual rank (MR) and highest reciprocal rank (HRR) correlation rank networks were constructed by summarizing all 1359 samples (33 experiments) as a whole. RNA-seq-derived correlation rank networks were retrieved from ATTED-II (https://atted.jp/) and CoNekT (https:// conekt.sbs.ntu.edu.sg/) plant gene co-expression database and from earlier studies (Wong et al. 2018; Sun et al. 2018, 2019; Vannozzi et al. 2018).
Network performance evaluation and gene function prediction

Networks were assessed for functional connectivity (performance) via GBA neighbor voting- and enrichment-based performance metrics. GBA neighbor voting-a machine learning algorithm based on the GBA principle-was performed in cross-validation across all given annotations and genes using the $E G A D$ R package with default settings (Ballouz et al. 2017). Each network was scored by the area under the receiver operator characteristic curve (AUROC) across MapMan BIN and Pfam functional categories using threefold cross-validation. MapMan BIN and Pfam annotations were limited to groups containing 20-1000 genes to ensure robustness and stable performance when using neighbor-voting (Ballouz et al. 2015; Verleyen et al. 2015).

Enrichment-based performance metrics were conducted as previously described (Ulitsky and Shamir 2009). Briefly, for each gene and all connected genes forming a group (i.e. subnetwork), multiple testing corrected enrichment P-values (false discovery rate, FDR) of MapMan BIN terms were first determined as previously described (Wong et al. 2016, 2017). Next, the fraction of terms enriched in at least one subnetwork at FDR $<10^{-4}$ (sensitivity) and the fraction of subnetwork enriched with at least one term at FDR $<10^{-4}$ (specificity) were determined and the overall performance score, F-measure was then calculated by taking the harmonic mean between sensitivity and specificity $[2 \times($ sensitivity $\times$ specificity $) /$ (sensitivity + specificity)].

\section{Functional term and cis-regulatory element enrichment analysis}

Enrichment of cis-regulatory elements (CREs) within each EXPANSIN gene subnetwork was performed as previously described with several modifications (Wong et al. 2016). Briefly, CREs inferred from the high-throughput TF binding site discovery method-the DAP motifs-were used (O'Malley et al. 2016). After the removal of redundant motifs, 404 DAP motifs encompassing 48 distinct TF families were retained and subjected to enrichment test considering the $1 \mathrm{~Kb}$ promoter region from the transcription start site and the + strand only. Multiple testing corrected enrichment P-values $($ FDR $)<0.05$ determines significantly enriched CRE in each EXPANSIN gene subnetwork while an FDR $<0.01$ determines significantly enriched BIN term (results obtained from the enrichment-based performance evaluation of the aggregate). 


\section{Results}

\section{The rationale of parameter settings in performance assessments}

Gene co-expression network performance is often sensitive to multiple parameters, either individually or in combination (Obayashi and Kinoshita 2009; Usadel et al. 2009; Ballouz et al. 2015; Serin et al. 2016; Obayashi et al. 2018). These include, but are not limited to the type of platform, correlation metric and thresholds used to establish co-expression, the type of gene annotation and functional ontology, and network evaluation metrics. In this study, two per-gene sparsity levels that are commonly adopted for gene function prediction and designing functional studies were considered. The top 100 and $300 \mathrm{co}-$ expressed genes (Obayashi et al. 2018), hereafter referred to as stringent and relaxed thresholds in this study, typically corresponds to the top $0.33-0.34 \%$ (stringent) and $1.00-1.04 \%$ (relaxed) of co-expressed genes in each gene list when using the Nimblegen whole-genome array and RNA-seq datasets with the widely adopted $12 \mathrm{Xv} 1$ gene annotation (29,970 genes).

For the choice of annotation scheme used for functional gene prediction and performance evaluation, we prioritized the MapMan BIN annotation (Thimm et al. 2004) over conventional ones (e.g. Gene Ontology, GO) for a few reasons: MapMan annotation is i. specifically designed for plant-specific processes and pathways, ii. contain less ambiguous terms that impede interpretation (e.g. enrichment results), and iii. was shown to be superior in gene function prediction performance compared to GO in GCN analysis (Thimm et al. 2004; Klie and Nikoloski 2012; Bolger et al. 2018). As an alternative reference for performance assessment, we will take advantage of the Pfam domain - a protein family- and domain-based functional identification scheme known for its comprehensiveness in ascribing many plant gene families (Bolger et al. 2018).

For the choice of metric used to assess performance, we prioritized the AUROC metric for its robustness but also its scalability across hundreds to thousands of functional terms and very large network sizes (i.e. $>10,000$ genes) (Ballouz et al. 2017).To ensure robustness and stable performance when using AUROC in GCN performance evaluations, functional annotations (e.g. GO) were often subsetted to the terms containing 20-1000 genes in human and yeast (Ballouz et al. 2015; Verleyen et al. 2015) and 20-300 genes in maize (Huang et al. 2017). In this study, only MapMan BIN and Pfam terms with 20-1000 annotated genes were used for AUROC performance evaluation. From a total of 1333 MapMan BIN terms (17,644 genes), only 285 terms (16,965 genes) satisfied this criterion. Similarly, of the 2927 Pfam terms $(17,954$ genes), 187 (8920 genes) were retained for evaluation.

To benchmark the robustness of our preferred reference annotation and performance metric choices, we assessed the informativeness of using functional enrichment analysis of gene sets across the underlying gene network to evaluate the performance of predicting functional terms (Ulitsky and Shamir 2009). This enrichment-based performance assessment will consider all 1333 MapMan BIN terms to evaluate the specificity, sensitivity, and F-measure performance of each network under investigation.

\section{Network performance across individual experiments}

To gain an understanding of how well each experimental project performed in our compendia prior to aggregation, 33 individual networks were first constructed with stringent (IndNet100) and relaxed (IndNet300) thresholds. Regardless of sparsification, the average AUROC performance across the corpus of individual experiments was only of modest performance, often AUROC $<0.7$ (Fig. 1a). For example, performance of BIN AUROCs across individual networks were clustered around an average of 0.62 (IndNet100) and 0.65 (IndNet300) while the Pfam AUROC were 0.60 (IndNet100) and 0.64 (IndNet300), respectively. A positive trend $\left(\mathrm{R}^{2}\right.$ of $\left.0.47-0.49\right)$ of improved performance was also observed with increasing sample sizes across experimental projects (Fig. 1b). For example, bIndNet300 networks constructed from large sample sizes ( $>100$ samples) in particular achieve close to or greater than AUROC 0.7. They include only two experimental projects-the grapevine expression atlas that captures the transcriptomes of diverse organs/tissues across a wide range of development (Fasoli et al. 2012) and the berry development transcriptome 'plasticity' study under various environmental and agricultural regimes (Dal Santo et al. 2013a). Conversely, networks constructed from small sample sizes ( $<20$ samples) often show poorer AUROC performance (AUROC 0.55-0.65).

Closer inspection of individual BIN and Pfam AUROCs revealed that some terms exhibit good performance (AUROC $>0.7$ ) across all individual networks, however, they make up a small proportion with $8-18 \%$ of the total terms evaluated fitting this criterion (Fig. 1c). Similarly, terms that showed exceptionally poor performance (AUROC $<0.55$ ) were also a relative minority, occupying $3-16 \%$ of the total terms evaluated. With a primary focus on BIN terms, we found that $32(11 \%)$ and $53(18 \%)$ of the 285 terms tested had average performances exceeding AUROC of 0.7 across IndNet100 and IndNet300 networks, respectively (Supplementary Table S1). These include terms related to photosynthesis (e.g. BIN1.1, BIN1.3), secondary metabolism (BIN16.8.2, BIN16.1.5), 
Fig. 1 Individual microarray gene co-expression network performance at predicting gene function. a The distribution of BIN and Pfam AUROC performance of 33 individual experiments at stringent (top100) and relaxed (top300) sparsity thresholds. Dashed lines indicate the average AUROC performance across all individual experiments at each sparsity threshold under evaluation. b The relationship of individual network AUROC performance with their underlying experimntal project sample size. Linear regression lines and $r^{2}$ values of each sparsity threshold under evaluation are depicted. c Heatmap of each BIN and Pfam term performance (ranked by descending mean term AUROC) across all individual experiments (ranked by ascending AUROC)
(A)

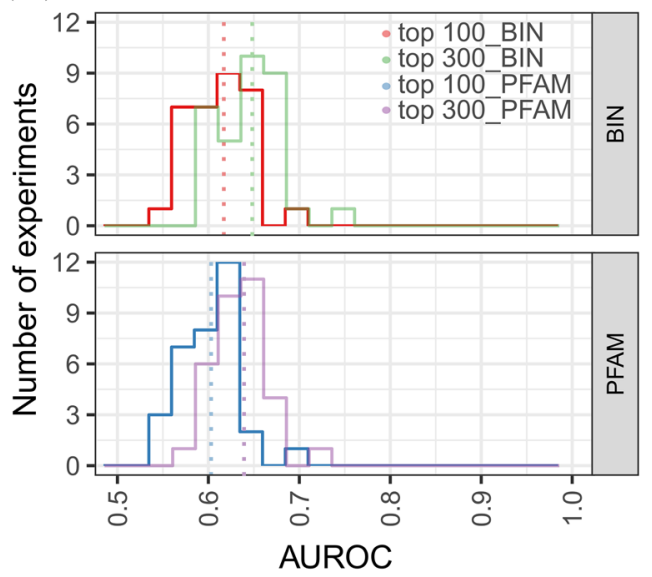

(B)

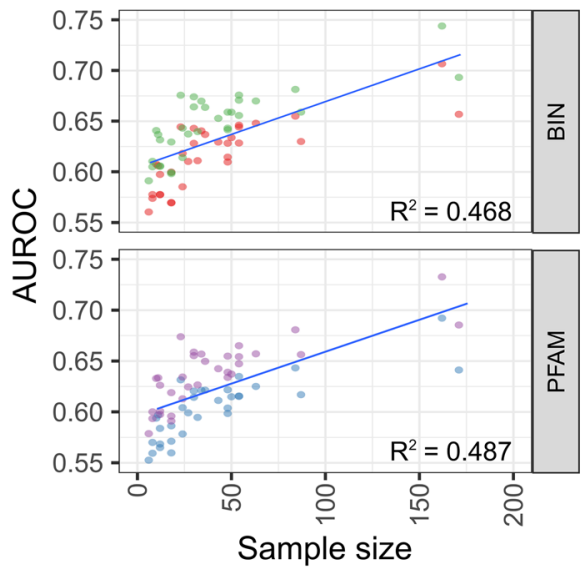

AUROC

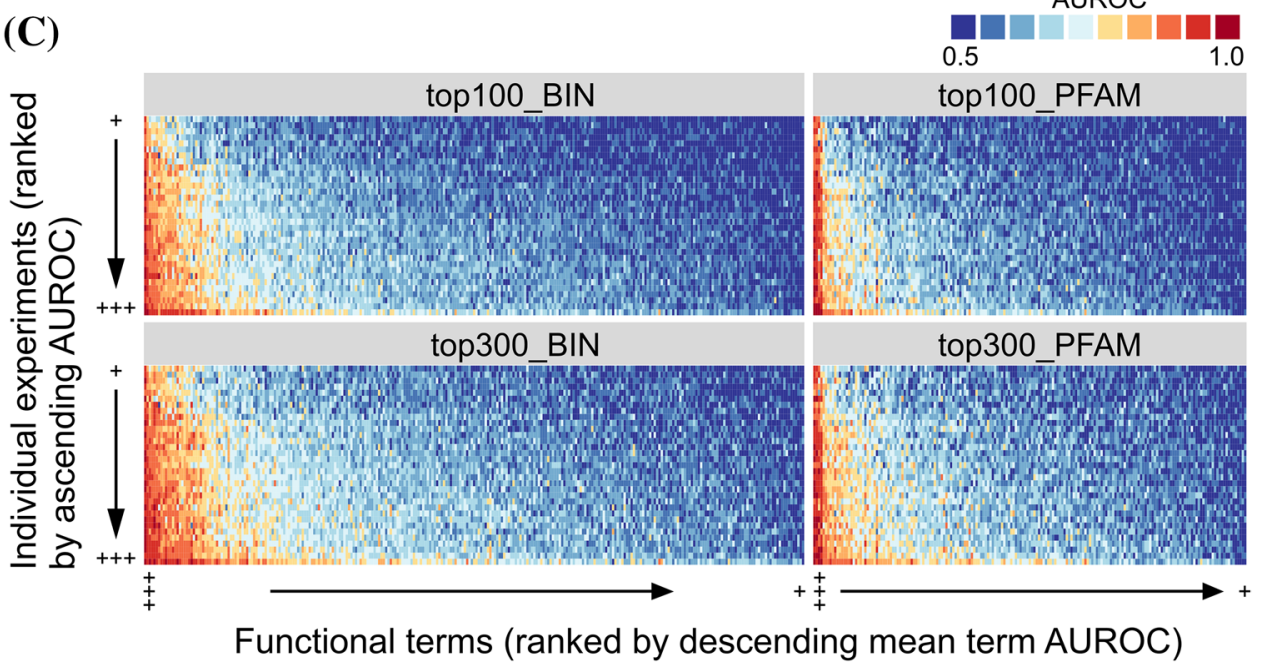

stress (BIN20.1.7, BIN20.2.1), and protein (BIN29.2), among others. Conversely, those that performed poorly (AUROC $<0.55$ ) include those within nucleotide metabolism (BIN23.1, BIN23.3), transport (BIN34.7, BIN34.14, BIN34.21), and several transcription factor families (BIN27.3.9, BIN27.3.20, BIN27.3.35), among others. Consistent findings were also observed with Pfam terms across IndNet100 and IndNet300 networks (Supplementary Table S1). For example, Pfam terms such as PF00195 (Chalcone and stilbene synthases), PF03936 (Terpene synthase family), PF00931 (NB-ARC domain), and PF00011 (Hsp20/alpha crystallin family) sharing strong correspondence with BIN16.8.2, BIN16.1.5, BIN20.1.7, and BIN20.2.1 respectively, were also shown to perform well (AUROC $>0.7$ ). Similarly, Pfam terms that showed exceptionally poor performance such as PF00293 (NUDIX domain), PF00999 (Sodium/hydrogen exchanger family), PF00083 (Sugar and other transporters), and PF07716 (Basic region leucine zipper), also have their related BIN counterparts BIN23.3, BIN34.14, BIN34.7, and BIN27.3.35, performing poorly.

\section{Aggregating across multiple networks improves network performance dramatically}

To investigate whether aggregating across multiple networks improves network performance, we aggregated individual networks in random order and perform this in a stepwise manner beginning with 2 until to 32 datasets/experiments. Briefly, in each iteration (i.e. addition of each network), the number of co-occurring interactions were used as edge weights, ranked in descending order, and thresholded at a per-gene sparsity of $0.34 \%$ and $1.04 \%$ (See Methods for details). Notably, our analysis clearly showed that aggregation across all 33 experiments improves performance dramatically as captured by BIN and Pfam (Fig. 2a). In all per-gene sparsity and annotation combinations tested, we observed that performance variability and gains decrease with increasing iterations. The first 14 iterations showed the largest performance variability but also the most gains (AUROC $>0.1$ ). In subsequent iterations, variability becomes minimal and performance gains have begun to plateau. Nonetheless, performance gains were still appreciable 
Fig. 2 Aggregate microarray gene co-expression network performance at predicting gene function. a The distribution of BIN and Pfam AUROC performance across random networks (2-32 experiments) and the full aggregate (33 experiments) at stringent (top100) and relaxed (top300) sparsity thresholds. At each iteration, aggregation curves were obtained from 10 random orderings of experiments. b Relationship of random aggregate sample size with increasing iterations. Linear regression lines and $r^{2}$ values are depicted. c The distribution of BIN and Pfam AUROC performance of the final aggregate. Dashed lines indicate the average performance across all BIN and Pfam terms at each sparsity threshold under evaluation
(A)

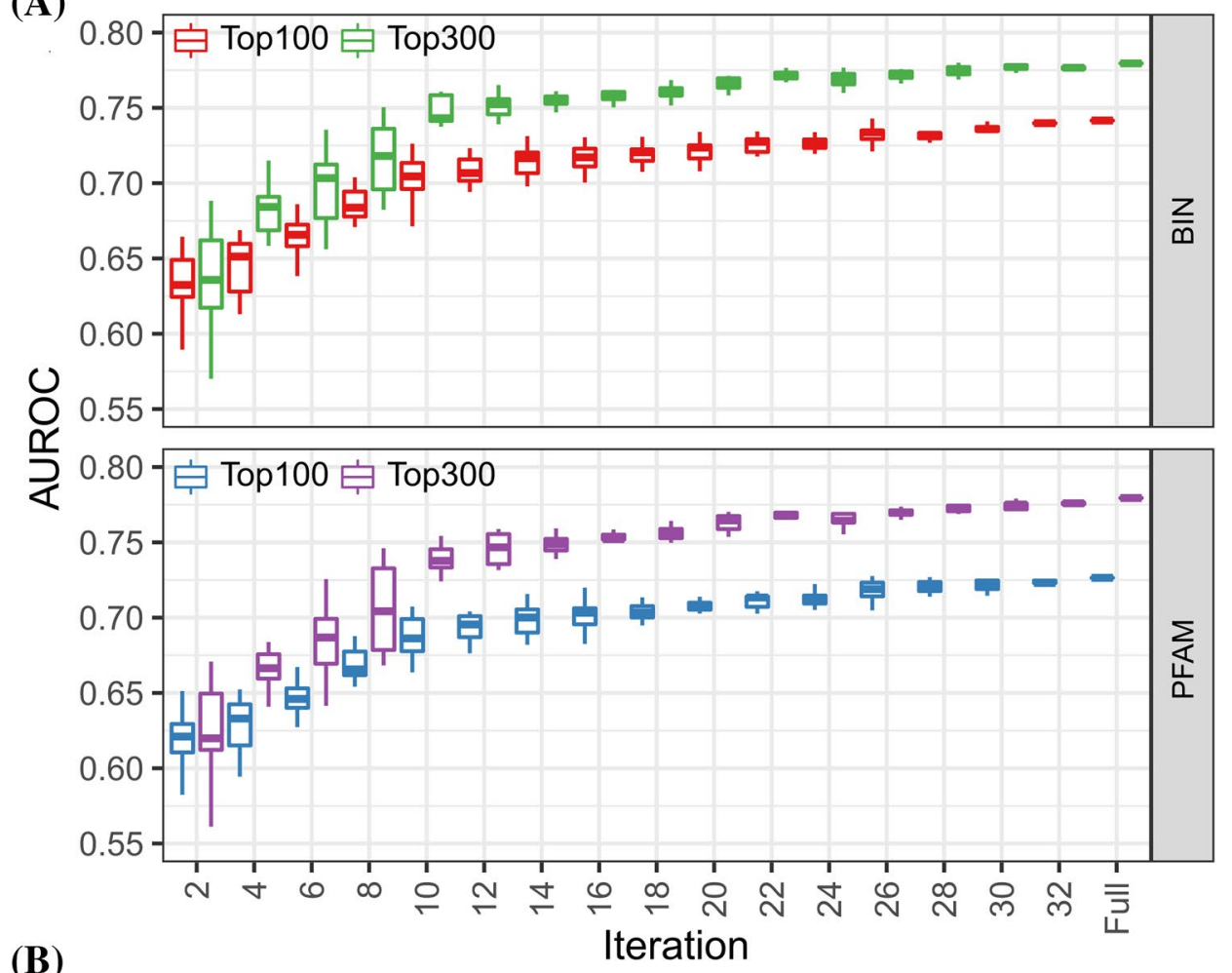

(B)

Iteration

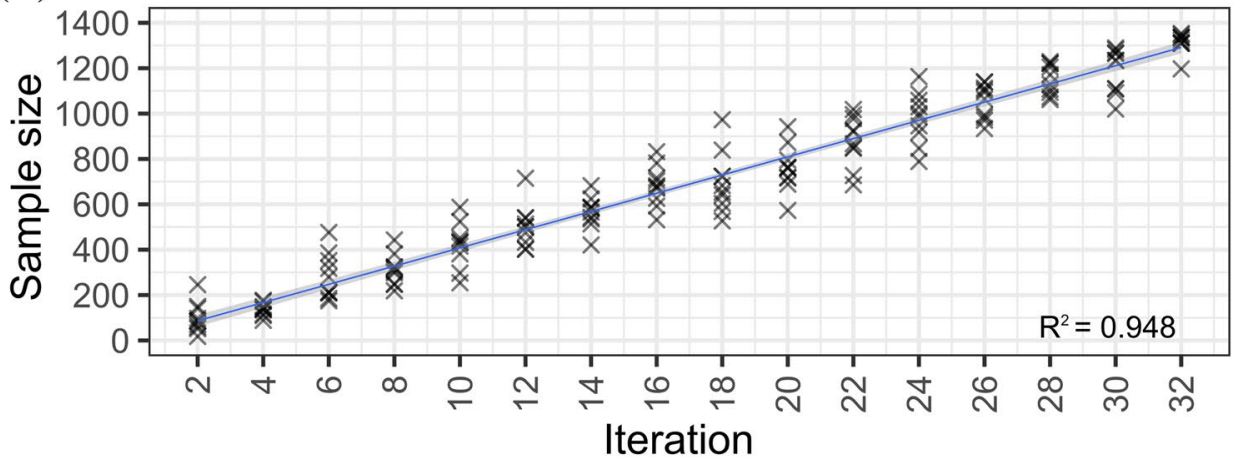

(C)
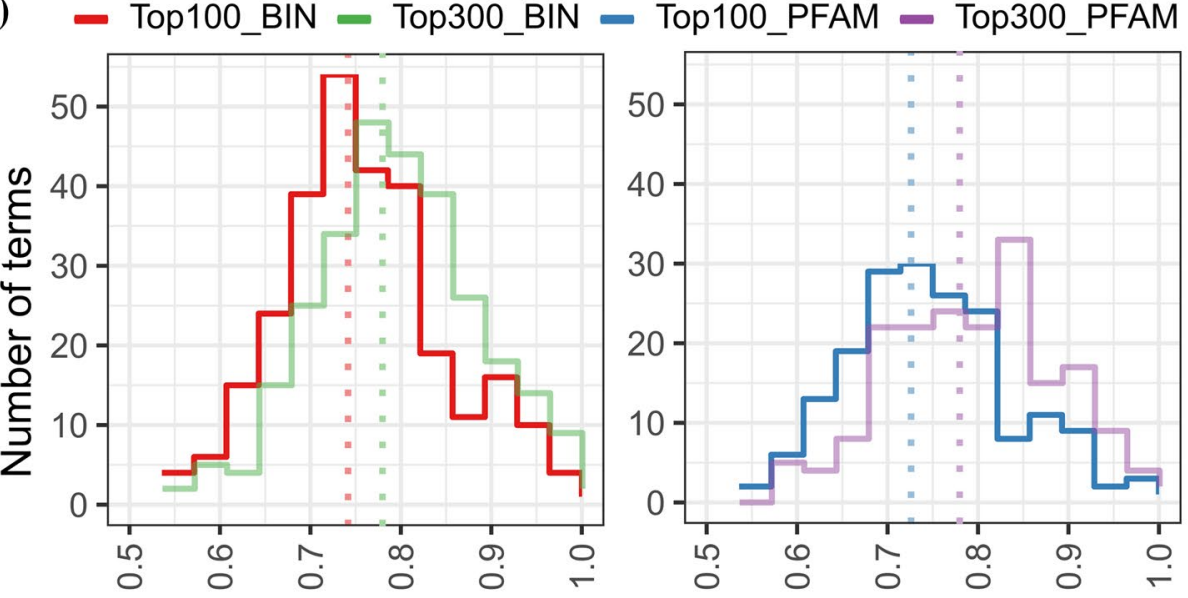

AUROC 
even when comparing them within this range (i.e. performance at iteration $20 \mathrm{vs} 30$ ). The total size of networks used for aggregation also scaled linearly $\left(R^{2}=0.948\right)$ with increasing iterations (Fig. 2b). The full aggregated network encompassing 33 datasets was of very good performance (Fig. 2c) surpassing the very few good-performing individual datasets in our assessments (Fig. 1b, Supplementary Table S1). The average BIN AUROC performance in the full aggregated network at $0.34 \%$ (AggNet100) and $1.04 \%$ sparsity (AggNet300) was 0.74 and 0.78 , while the Pfam AUROC were 0.73 and 0.78 , respectively.

Considerable mean performance improvement of $\sim 0.13$ was observed when comparing the aggregate to the averages across individual (non-aggregated) networks (Fig. 3a). Dramatic performance gains (AUROC $>0.1$ ) were often observed with terms that did not perform well across individual networks (average AUROC $<0.7$ ). In the aggregate, these terms clustered around an AUROC of 0.65-0.80 (AggNet100) and 0.7-0.85 (AggNet300) respectively (Fig. 3b). Conversely, terms that performed well in individual networks (AUROC $>0.7$ ) often show depreciating performance gains from aggregation (Fig. 3a). Furthermore, the majority of annotations (188 of 285, 66\%) never outperform the aggregate network across any individual network (Supplementary Figure S1). However, terms that often do, show lower performance on average (AUROC $<0.65$ ) in both the aggregate and across individual networks. We repeated these series of tests using Pfam annotations and found consistent findings.

With an emphasis on BIN terms, we observed that $67 \%$ (191 of 285) and 80\% (233 of 285) of total terms have an AUROC $>0.7$ in the AggNet100 and AggNet300 networks, respectively. In comparison with the individual networks, 189 additional terms have an AUROC $>0.7$ in the aggregate (Supplementary Table S1). Of these, 128 BIN terms are in common, while 9 and 52 are unique to AggNet100 and AggNet300, respectively. Terms that shown the most dramatic gains (AUROC $>0.15$ ) are also plentiful and diverse in the aggregate- 72 and 121 terms in AggNet100 and AggNet300, respectively. Among others include those that belong to amino acid metabolism (BIN13.2), secondary metabolism—wax (BIN16.7), lipid metabolism (BIN11.1), cell wall metabolism (BIN10.1, BIN10.5), hormone metabolism (BIN17.7), and TCA/organic acid transformation (BIN8.1) pathways. Notwithstanding the widespread performance boost seen in the aggregate networks, there were still a few $(<10)$ terms that still perform poorly (AUROC $\sim 0.55$ ) and do not benefit substantially with aggregation (Supplementary Table S1). Nonetheless, there was no parent/toplevel BIN category showing consistently poor predictive
(A)
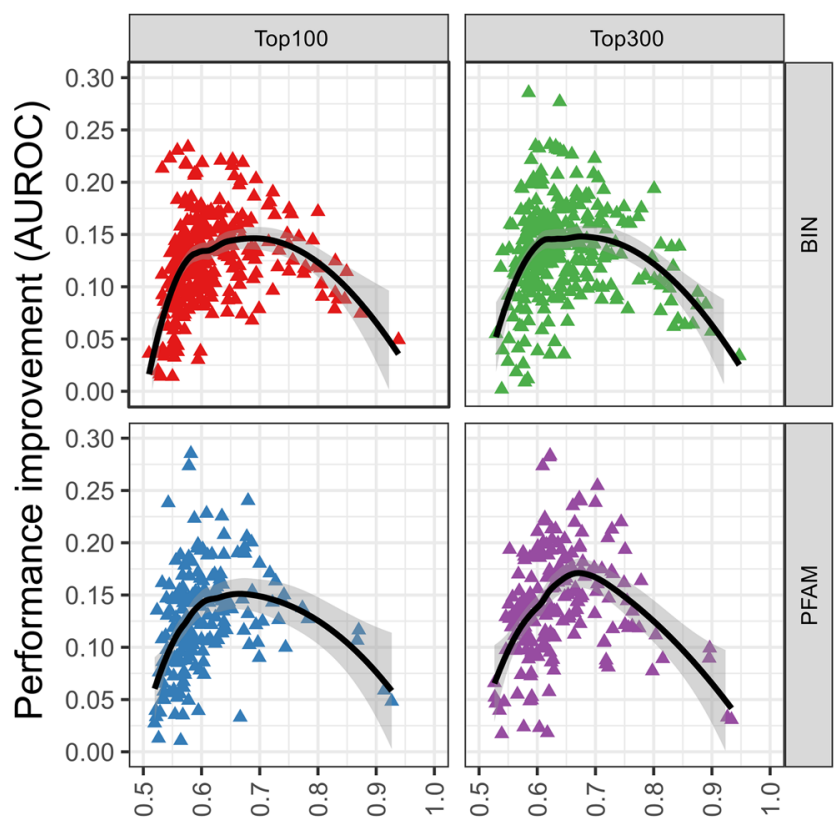

averaged AUROC performance across individual networks

Fig. 3 Properties of performance improvement in the aggregate gene co-expression network. Distribution of each BIN and Pfam AUROC performance improvement curves relative to a the average term
(B)

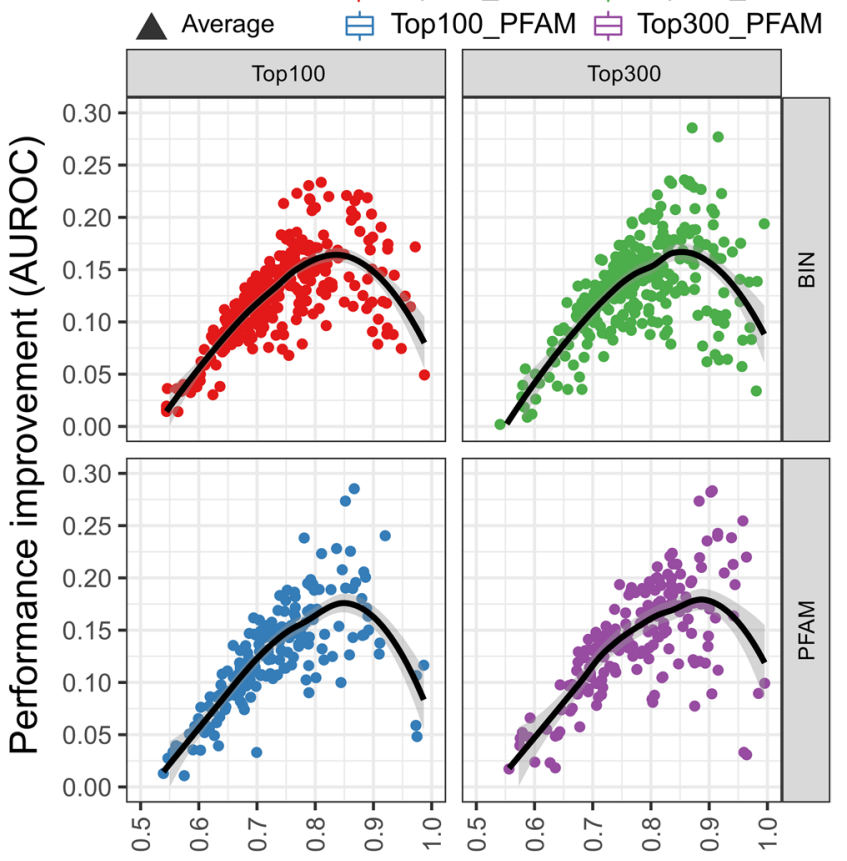

AUROC performance in the aggregate

AUROC performance across individual networks and $\mathbf{b}$ the final aggregate network at stringent (top100) and relaxed (top300) sparsity thresholds 
performance except for nucleotide metabolism (BIN23) at stringent thresholds. Repeating these series of tests using Pfam annotations also revealed consistent trends (Supplementary Table S1).

The relationship of sparsification on i) the co-expression frequency edge weights and ii) their underlying PCC values in the were also investigated. We found that the median co-expression frequency in the AggNet 100 and AggNet300 network was 5 and 4 occurrences, respectively (Supplementary Fig. 2). The majority of these co-expression links were characterized by strong positive PCC values, having a median PCC of 0.77 and 0.74 , respectively (Supplementary Fig. 2). In comparison, at extreme sparsification thresholds $(\sim 0.001 \%)$, the median occurrence and PCC were slightly higher between 8-9 occurrences and 0.8-0.82 PCC, respectively. Furthermore, a minimal overlap in PCC distribution between the observed and random aggregate (i.e. derived from aggregating individual experiments whose expression was fully randomized) in both stringent and relaxed sparsity thresholds further substantiates the robustness of the aggregate.

\section{Comparative study of the aggregate with other state-of-the-art microarray and RNA-seq networks}

In the last decade, the grapevine Nimblegen whole-genome array and recently, RNA-seq transcriptomes provided the bulk majority of datasets amendable for conventional (nonaggregate) GCN construction in grapevine (e.g. Wong et al. 2017; Obayashi et al. 2018; Proost and Mutwil 2018; Vannozzi et al. 2018). Most often, large gene expression matrices (often hundreds to thousands of samples) and the rank of correlation were used to construct their corresponding correlation-rank GCNs directly without the need for aggregating multiple GCNs. As the rank of correlations also permits equivalent performance comparisons at the same per-gene sparsity threshold across different co-expression data (Obayashi and Kinoshita 2009), we ask whether the aggregated network provide any appreciable gains (or none at all) in functional performance against a variety of microarray- and RNA-seq-derived correlation rank GCNs. In this study, we considered six distinct scenarios for performance comparison: Three microarray-derived GCNs constructed by summarizing all 1359 samples (33 experiments) as a whole (this study)-1. PR (micro_PR), 2. highest reciprocal rank (micro_HR), and 3. mutual rank (micro_MR) - and three RNA-seq-derived GCNs. The latter includes a 4. MR GCN (rnaseq1_MR) used in four recent reports (Wong et al. 2018; Sun et al. 2018, 2019; Vannozzi et al. 2018), a 5. HRR GCN (rnaseq2_HR) available from CoNekT (https://conekt.sbs. ntu.edu.sg/), and a 6. MR GCN (rnaseq3_MR) available from ATTED-II (https://atted.jp/) plant gene co-expression database. These RNA-seq GCNs were constructed from 654
(rnaseq1_MR), 612 (rnaseq2_HR), and 346 (rnaseq3_MR) RNA-seq datasets, respectively.

Performance rankings of all seven networks evaluated were highly congruent across the two sparsification threshold and two references tested. The AUROC performance was generally good (average AUROC $>0.7$ ) especially with relaxed sparsification, however, none outperformed the aggregate (Fig. 4, Supplementary Figure S3). The majority of BIN (51-74\%) and Pfam (44-71\%) terms showed good performance $(A U R O C>0.7)$ in the microarray-derived networks (Supplementary Figure S4 and Table S2). Conversely, good-performing terms in RNA-seq-derived networks were much lower (27-66\% of BIN and $29-56 \%$ of PFAM terms). We observed that the AUROC performance of all three rank of correlation metrics (i.e. micro_PR, MR, and HR) assessed using whole microarray datasets were very similar, with minimal variation among them. In addition, we observed that microarray-derived networks (the aggregate and non-aggregate) generally perform better than those constructed with RNA-seq datasets. Similarly, the aggregate (i.e. at iterations 8 and 16) and microarray correlation rank networks (i.e. at $\sim$ subset size of 250 and 650 ) downsampled to that of corresponding RNA-seq network sizes consistently showed superior performance (Fig. 2a, b, Supplementary Figure 5). Nonetheless, of the three RNA-seq networks evaluated, rnaseq1_MR was the only one that comes closest to the performance indices of the aggregate or microarray correlation-rank networks.

Next, we explored in greater detail the performance gains of functional terms in the aggregate compared to the six networks evaluated. Across the comparisons, the performance of most terms ( $>60 \%$ ) never outperform the aggregate (Supplementary Figure S6 and Figure S7). For example, when the aggregate was compared to the microarray-derived networks, $64-80 \%$ of total terms had higher performance, with the majority showing slight gains (AUROC of 0.015-0.035) across a broad range of high- and low-performing terms. Conversely, when compared with the RNA-seq-derived networks, not only were there a higher proportion of terms (78-90\%) that are better-performing in the aggregate, strong gains in performance were often observed (AUROC $>0.05$ ). Nonetheless, terms that show noticeably stronger predictive performance (AUROC $>0.05$ ) in networks other than the aggregate, were often sporadic, albeit occurring more frequently in the RNA-seq networks (Supplementary Figure S8 and Table S3).

\section{GBA-based neighbor voting is robust and consistent with enrichment-based performance assessments}

In this study, we wanted to understand how our extensive assessments with GBA-based neighbor voting might generalize broadly. Using a GBA-independent, functional 
Fig. 4 Comparative analysis of the aggregate network performance with other microarray- and RNA-seq-derived co-expression networks. Violin plots depict the distribution of AUROC a BIN and b PFAM performance of various microarray (micro_PR, micro_MR, micro_HR)- and RNA-seq (rnaseq1_MR, rnaseq2_HR, rnaseq3_MR)-derived networks at stringent (top100) and relaxed (top300) sparsity thresholds. Points indicate the average AUROC performance across all functional terms for each network at respective sparsification

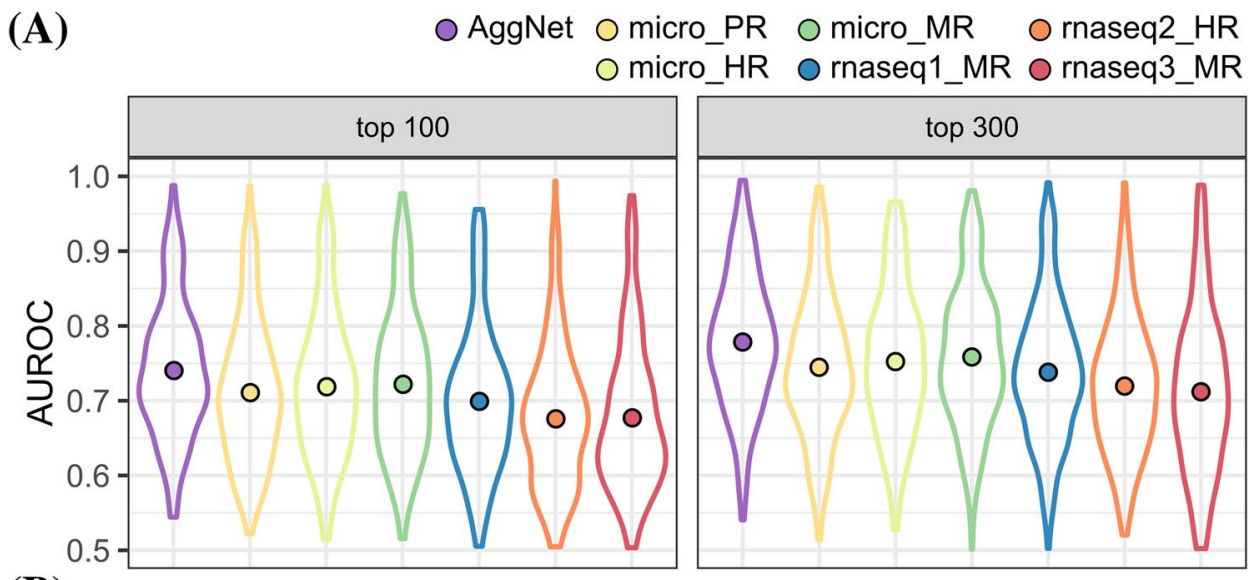

(B)

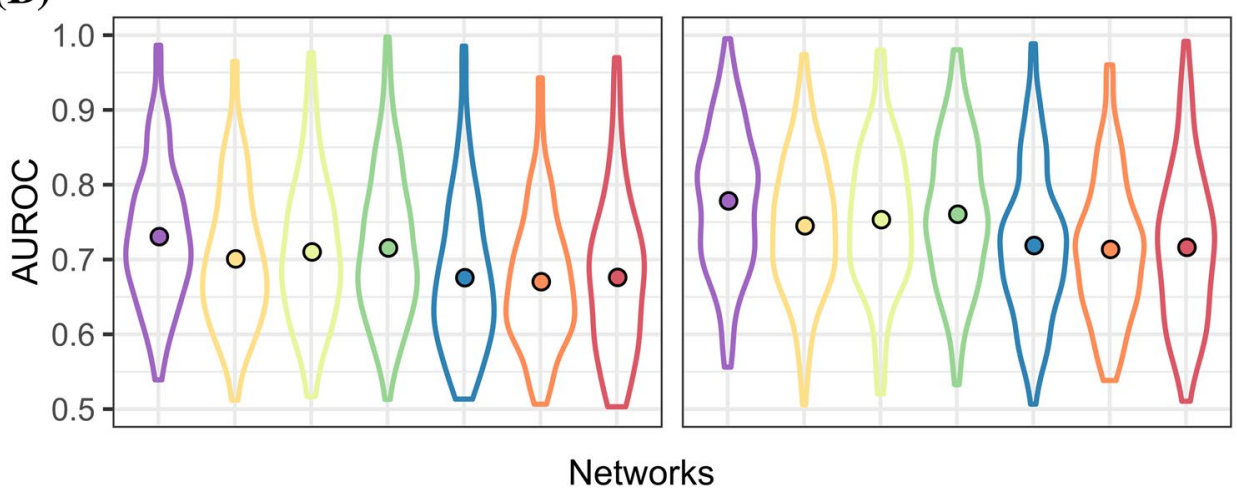

enrichment-based performance metric, we recalculated the performance of individual networks, random aggregate networks (2-32 datasets), the full (33 datasets) aggregated network, and six microarray- and RNA-seq-derived networks. As consistent findings were observed between BIN and Pfam terms in GBA-based performance assessments, we prioritize the former in this reevaluation. The BIN F-measure performance of individual networks at stringent and relaxed sparsification was highly congruent with their corresponding AUROC scores ( $\mathrm{R}^{2}$ of 0.93) (Fig. 5a, Supplementary Table S4). Interestingly, sensitivity was more highly correlated with AUROC performance $\left(\mathrm{R}^{2}\right.$ of $\left.0.96-0.97\right)$ while specificity was much poorer $\left(\mathrm{R}^{2}\right.$ of $\left.0.71-0.82\right)$. Nonetheless, both methods demonstrated highly consistent rankings across high- to low-performing experimental projects. In the random and full aggregate networks, performance trends remained surprisingly congruent across both metrics (Fig. 5b). Notably, aggregate networks constructed from a small number of datasets (i.e. 2-14) showed large variability in BIN F-measure performance. Subsequent addition of datasets greatly improved performance stability, however, performance gains become minimal (or plateau) from $>28$ datasets. Upon closer inspection, we also observed that sensitivity was remarkably consistent across strict and relaxed sparsification while specificity was notably higher in the latter across the range of aggregates.
Enrichment-based performance assessments also scaled linearly with AUROC scores across the aggregate and other microarray- and RNA-seq-derived networks and highlighted the superiority of the aggregate in many aspects (Fig. 5c). Dramatic performance gains were observed especially when comparing the aggregate against the rnaseq2_HR and rnaseq3_MR networks (F-measure improvement of $0.1-0.13$ ) while appreciable gains were still seen against the others (F-measure improvement of 0.01-0.03). In terms of sensitivity, none of the networks evaluated outperform the aggregate (Fig. 5d). When compared to the next best and weakest performer, aggregate achieved 1.10-1.2 and 1.71-1.84 fold higher sensitivity, respectively. In terms of specificity, no single network emerged as a consistent top-performer. When compared to rnaseq1_MR and rnaseq3_MR, the other two best performing networks in terms of specificity, the aggregate had just 1.03-1.15 $\times$ lower specificity. Thus, the higher performance of the aggregate may be attributed to an overall improvement in sensitivity as the specificity scores are comparable across the other networks evaluated.

\section{Example application: the grapevine expansin gene superfamily}

Expansins (EXPs) are cell wall loosening enzymes implicated in the regulation of cell wall expansion and 
(A)

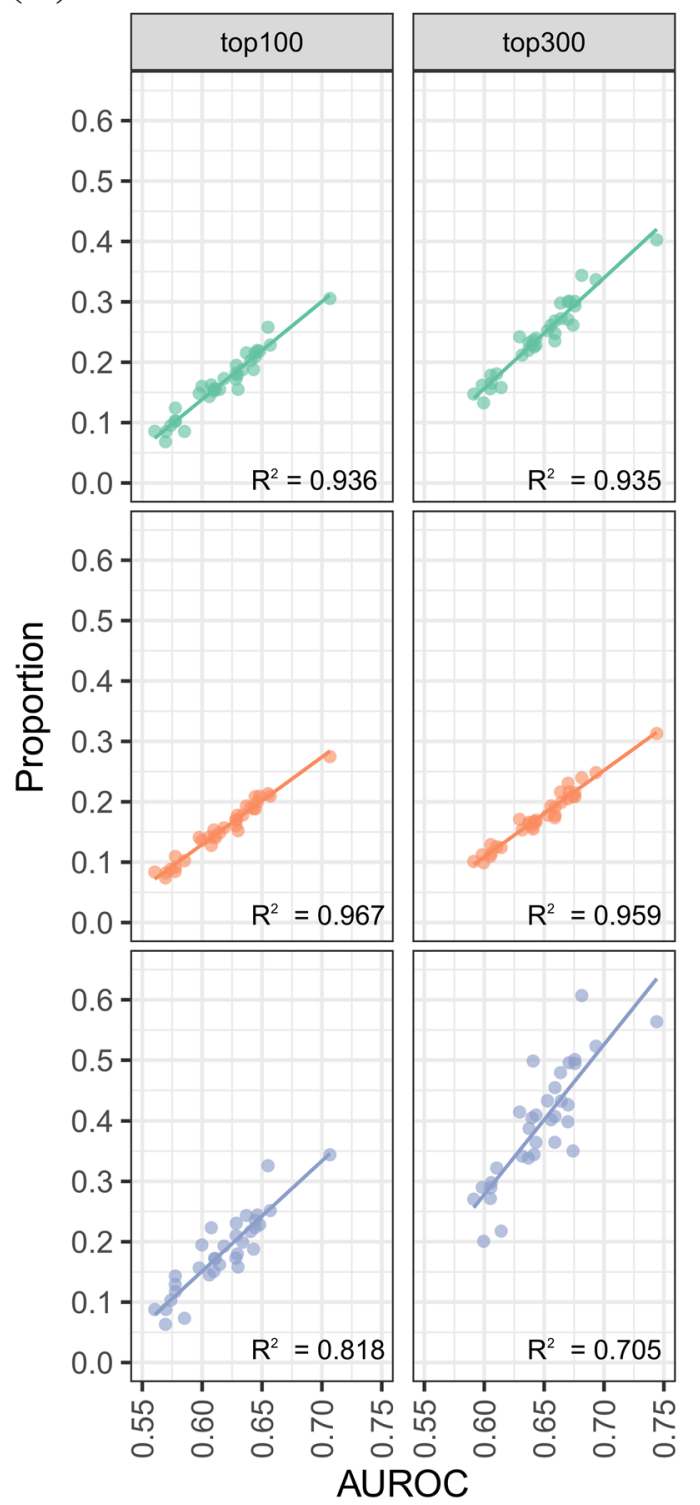

(C)

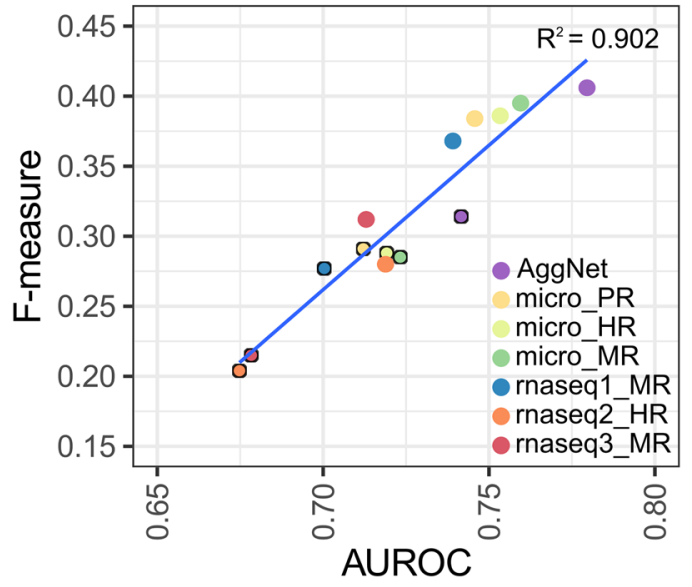

(B)

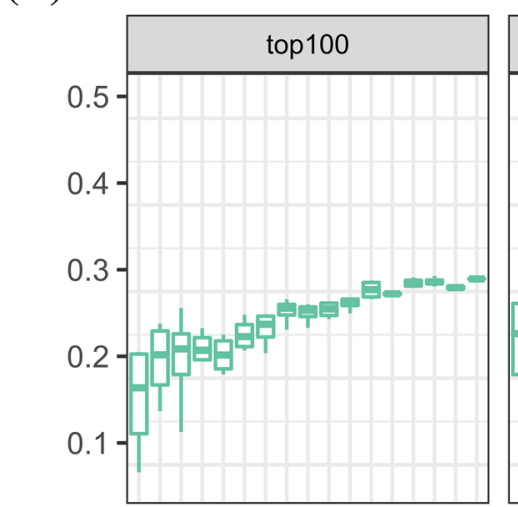

- Sensitivity - Specificity
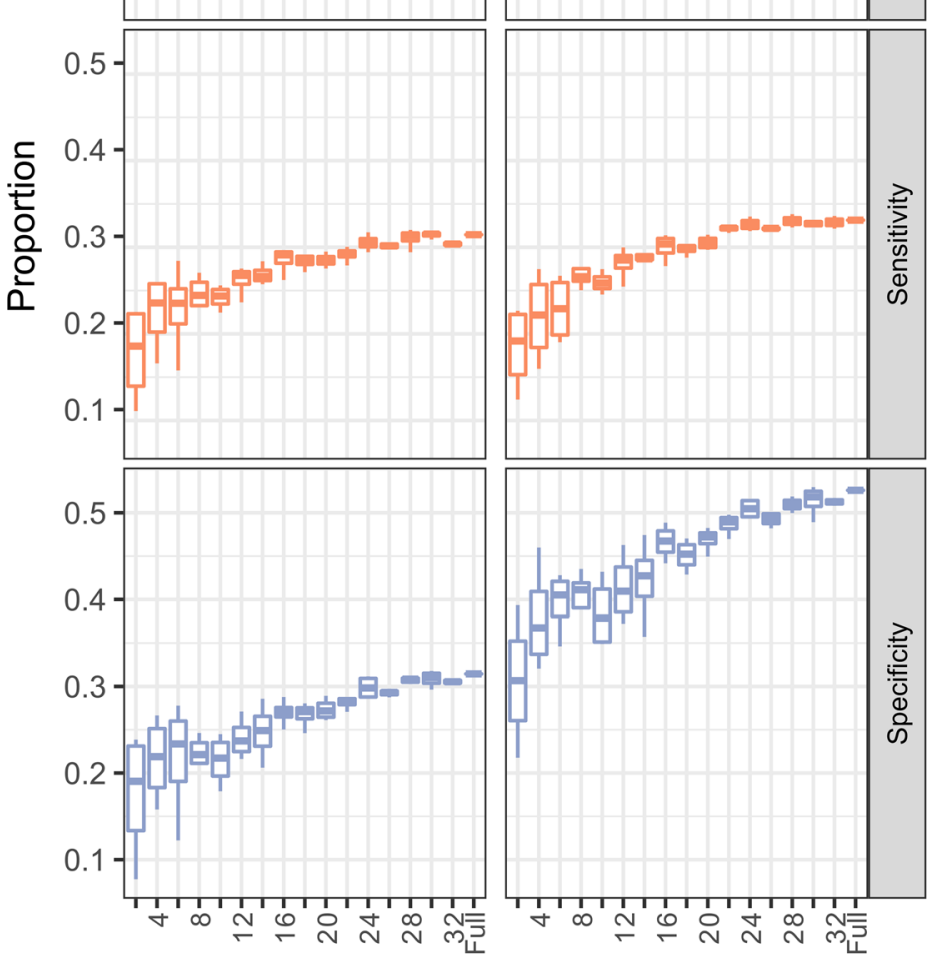

Iteration

(D) $\bigcirc$ AggNet $O$ micro_PR $O$ micro_MR O rnaseq2_HR O micro_HR O rnaseq1_MR o rnaseq3_MR

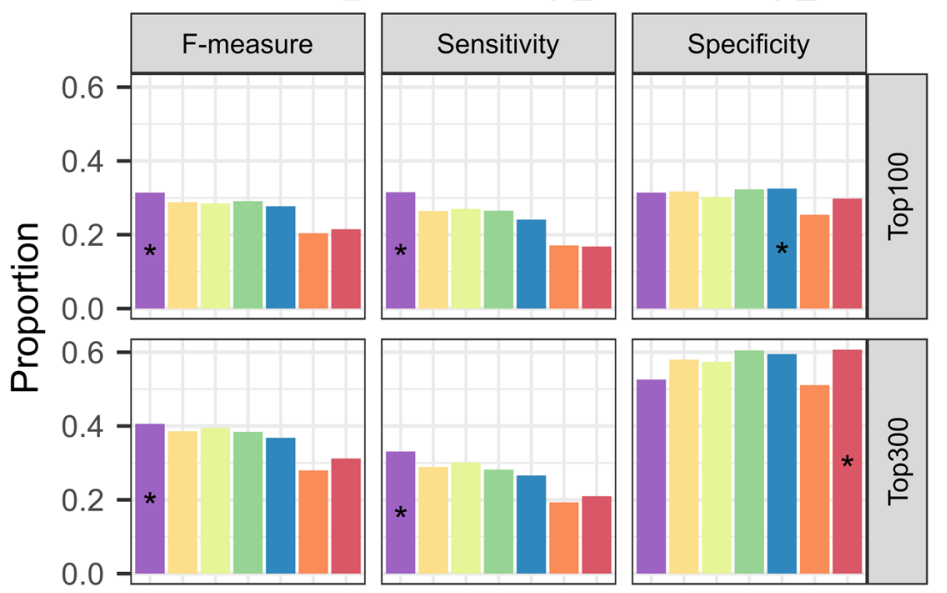


४Fig. 5 Comparative analysis of guilt-by-association neighbor voting and enrichment-based performance at predicting function. a The relationship of sensitivity, specificity, and F-measure with AUROC BIN performance across 33 individual experiments at stringent (top100) and relaxed (top300) sparsity thresholds. b The distribution of sensitivity, specificity, and F-measure BIN performance across random networks (2-32 experiments) and the full aggregate (33 experiments). At each iteration, aggregation curves were obtained from 10 random orderings of experiments. $\mathbf{c}$ The relationship between F-measure and AUROC BIN performance (linear regression lines and $r^{2}$ values are depicted) and $\mathbf{d}$ underlying sensitivity and specificity scores of the aggregate and other microarray- and RNA-seq-derived networks. Circles with and without the black border in $\mathbf{c}$ depict F-measure at stringent and relaxed sparsity thresholds, respectively

enlargement in a pH-dependent manner (Cosgrove 2015). In plants, there are four distinct EXP families-expansin A (EXPA), expansin B (EXPB), expansin A-like (EXLA), and expansin B-like (EXLB). Plant EXPAs and EXPBs are known to be involved in cell wall expansion and modification while the exact functions of EXLAs and EXLBs remain to be ascertained. In grapes, only two of the 29 predicted EXPs have been functionally validated (VvEXPA14 and VvEXPA18). In this study, we attempt to explore the potential functions of grape EXPs and their regulatory control by interrogating the subnetworks within AggNet300 (Supplementary Table S5) and determining what functional categories (Supplementary Table S6) and cis-regulatory elements (Supplementary Table S7) were enriched.

EXP subnetworks were often enriched with multiple distinct functional categories (Fig. 6, Supplementary Table S6). A summary depicting enriched functions restricted to highlevel BIN categories (depth $\leq 1)$ within EXP subnetworks showed that enrichment for cell wall (BIN10), miscellaneous enzyme reactions (BIN26), secondary metabolism (BIN16), and transport (BIN34) are among the most common. Within the enriched cell wall (BIN10) category, four EXP subnetworks (VvEXPA6, VvEXPA11, VvEXPA16, and VvEXPA18) were enriched in cell wall degradation (BIN10.6), modification (BIN10.7), and pectin \& esterases (BIN10.8) categories while six subnetworks (i.e. VvEXLA1, VvEXPA1, VvEXPA7, VvEXPA13, VvEXPA14, VvEXPA17) were enriched with two terms (i.e. BIN 10.6 and 10.7).

Many other enriched functional categories that may be of potential biological relevance were also found. For example, nine EXP subnetworks (7 EXPA and 2 EXPB) were enriched with photosynthesis-related light reaction terms (BIN 1.1). Four EXP subnetworks (VvEXPA5, VvEXPA7, VvEXPA11, and VvEXPA18) were enriched with transport-related major intrinsic proteins (BIN 34.19). Four EXP (VvEXLB2, VvEXPA7, VvEXPA8, and VvEXPB2) and three (VvEXPA8, VvEXPB1, and VvEXPA19) EXP subnetworks were enriched with flavonoid (BIN16.2) and phenylpropanoid (BIN16.8) pathway genes, respectively.
Two (VvEXLB2 and VvEXLB4) and two (VvEXPA11 and VvEXPA18) EXP subnetworks were also enriched with cytokinin (BIN 17.4) and brassinosteroid (BIN 17.3) hormone metabolic pathway genes, respectively.

We also compared the enriched functional categories of EXP duplicate pairs. We reanalyzed the genomic structure of EXP genes in grapevine using MCScanX (http://chibb a.pgml.uga.edu/mcscan2/) and found 9 EXP genes showing signatures of gene duplication. Proximal duplication events were detected for VvEXLB2-VvEXLB3 pair and VvEXPB3VvEXPB4 pair while WGD/segmental duplication events were detected for VvEXPA1-VvEXPA16-VvEXPA17 pair and VvEXPA6-VvEXPA11-VvEXPA13 pair. We found that subnetworks of WGD/segmental duplicated EXP genes tend to share a higher proportion of overlapping enriched categories (e.g. VvEXPA6 and VvEXPA11, VvEXPA16 and VvEXPA17) compared to the ones found in proximal duplicated EXP subnetworks whereby functional categories seem to have diverged (e.g. VvEXLB2 and VvEXLB3).

To dissect the potential upstream transcription factors regulating EXP subnetworks, we investigated the frequency of TF occurrence(s) and tested for enrichment for known CREs (Supplementary Table S7). We found that EXP subnetworks contain a diversity of binding sites from several TF families (Supplementary Figure S9A). However, some TF families were frequently present and have their corresponding CREs significantly enriched (FDR $<0.05)$ in many EXP subnetworks. They include the homeodomain-associated leucine zipper (HB), zinc finger-homeodomain, AP2/ERF, bZIP, bHLH, and NAC TF families, among others (Supplementary Figure S9B). For example, the CACGTG CRE, also known as G-box, commonly bound by bZIP and bHLH TFs, was the most frequently enriched CRE in EXP subnetworks. The CACGTG CRE was enriched in 1 EXLA, 2 EXLB, 7 EXPA, and 1 EXPB subnetwork(s) and coincides with a high frequency of bZIP and bHLH TFs in them. Similarly, the enriched AP2/ERF CREs (e.g. RCCGACA) were present in 1 EXLA, 5 EXPA, and 1 EXPB subnetwork(s) while enriched NAC CREs (e.g. DVCGTR, WRCTTG) were found in 1 EXLA, 3 EXLB 3 EXPA, and 3 EXPB subnetwork(s). Both AP2/ERF- and NAC-enriched subnetworks also contained the corresponding TFs in them.

\section{Discussion}

Large transcriptome datasets with experiments or samples numbering in the hundreds or thousands have enabled network aggregation across multiple experiments in both model plants (Liesecke et al. 2019) and in non-plant systems (Gillis and Pavlidis 2011; Ballouz et al. 2015; Verleyen et al. 2015). In these studies, thorough assessments of network performance under various settings that broadly generalize 


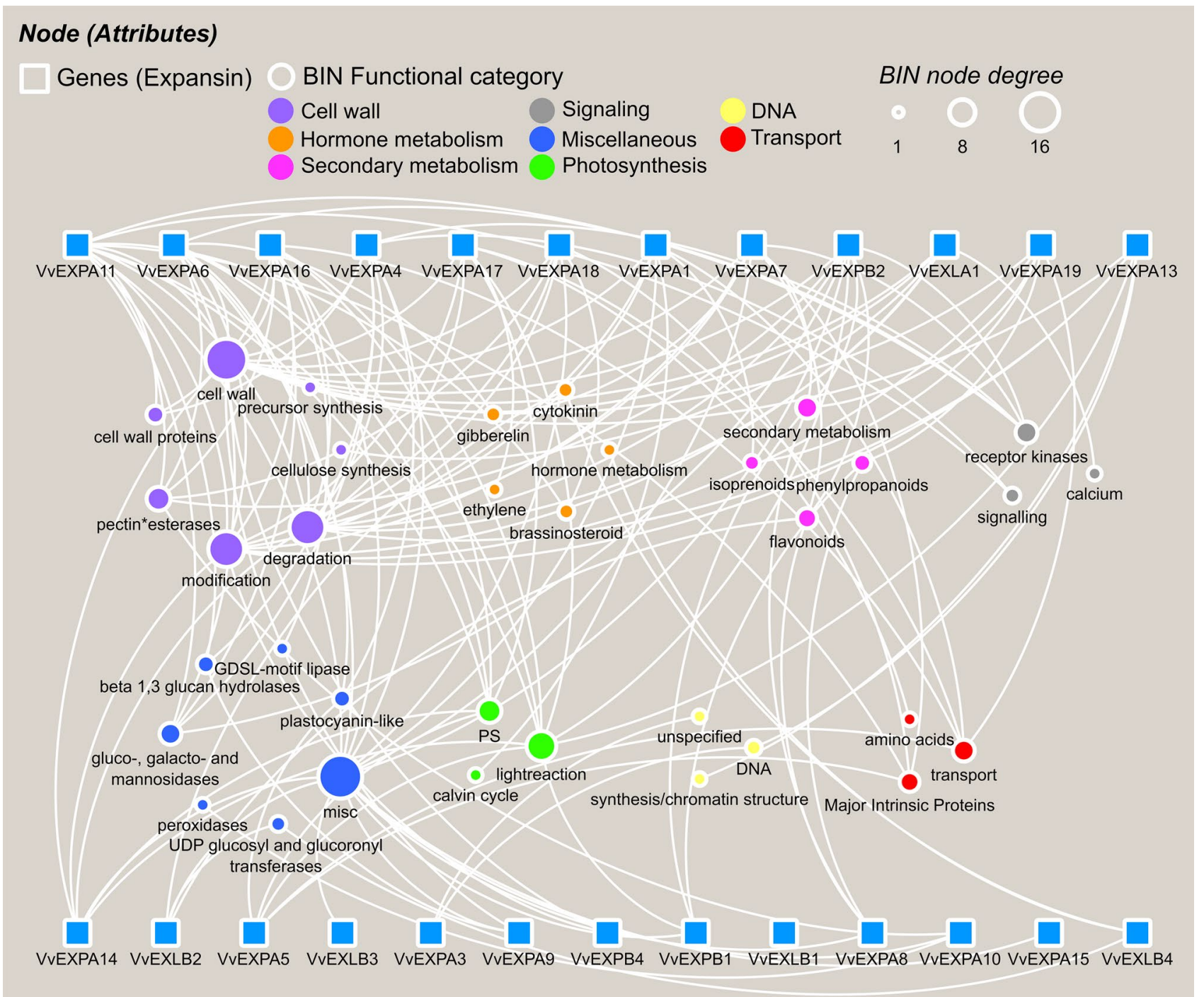

Fig. 6 Enriched functional categories of expansin gene co-expression networks. Square and circles nodes depict genes (expansin) and BIN functional categories, respectively. Filled color of circle nodes depicts the associated BIN categories (BIN depth $\leq 1)$. Circle size represents the frequency of expansin gene co-expression networks enriched with the corresponding BIN category. White edges connect each BIN category with their relevant EXP gene co-expression networks they are enriched in has also been undertaken. One key (and consistent) finding in these studies is that aggregating across multiple networks (e.g. using a simple rank-and-summate strategy) improves network performance dramatically as assessed by GBAbased neighbor voting.

In this study, we found that performance was generally poor (average AUROC $<0.65$ ) regardless of sparsity across the corpus of 33 individual networks (Fig. 1a, b). In comparison, performance gains were dramatic $($ AUROC $>0.1$ ) when individual networks were aggregated (Figs. 2, 3). In this study, aggregating across 14 separate experiments was already sufficient to obtain a high-quality network. As with other aggregate co-expression networks (Gillis and Pavlidis 2011; Ballouz et al. 2015), our evaluation suggests that we may be reaching a performance ceiling with microarray datasets. Although additional datasets still yield better performance (rather than diminishing them), minimal gains were observed when the number of aggregated networks exceeded 30 experiments. Our findings, and others, reinforce the need for having a modest quantity of data, i.e. many experiments and samples, to obtain reliable functional information when performing co-expression studies, especially with aggregation (Gillis and Pavlidis 2011; Ballouz et al. 2015; Liesecke et al. 2019).

Although secondary to the key findings of this study, we also found a dependency of performance on network sparsity. Using our choice of stringent and relaxed sparsification thresholds, we found that an increase in sparsity decreases 
network performance. It is well-known that sparsification affects network performance (Gillis and Pavlidis 2011; Liesecke et al. 2019). Networks constructed from information-free matrices (i.e. when sparsification are either too high or too low) often result in poorer performance compared to a determined optimum, however, finding this balance is not trivial (Gillis and Pavlidis 2011). While finding this optimum is favorable, our choice of sparsification has yielded networks of robust performance (average AUROC $>0.7$ ) but also within realistic means for designing functional studies of prioritized candidates (Obayashi et al. 2018). Similar sparsification thresholds have also been used in several systems-oriented grape functional studies with much success (Loyola et al. 2016; Sun et al. 2018; Vannozzi et al. 2018).

Regardless of sparsification, we also found that the aggregate network consistently outperforms a variety of microarray- and RNA-seq-derived correlation rank (non-aggregate) GCNs (Fig. 4, Supplementary Figure S6 and S7). In our evaluations, summarizing all 1359 samples from 33 experiments as a whole (i.e. micro_PR, HR, and MR) showed good performance, however, the aggregate still performed better. Nonetheless, our findings suggest that correlation rank GCNs are robust and informative, but can certainly benefit from the use of aggregation approaches. Interestingly, we found that RNA-seq-derived GCNs showed consistently poorer performance compared to both aggregated and nonaggregated microarray-derived GCNs in many aspects. One of the several straightforward reasons may be due to i. the type of annotations used and ii. the lack of sufficient RNAseq datasets used-in both the number of samples and its heterogeneity/diversity (i.e. types of experiments). Methodical variations introduced early on during the construction of RNA-seq transcriptome compendia alone may also be a contributing factor. These include how the RNA-seq reads were mapped (e.g. read depth constraints) and the type of normalization used (e.g. raw counts, FPKM, TPM, VST), among others (Giorgi et al. 2013; Ballouz et al. 2015; Huang et al. 2017).

While the micro-(PR, MR, and HR) and rnaseq1_MR GCNs conformed to the latest V1 annotation, the rnaseq2 HR and rnaseq3_MR networks make use of the depreciated NCBI gene (LOC_) and 12xV0 (GSVIVT_) gene prediction that represents $\sim 63 \%$ and $87 \%$ of genes relative to the latest $12 x V 1$ annotation (29,970 genes), respectively. Thus, function performance in the latter two networks may be compromised due to the reduced number of genes and functional terms that can be effectively compared. However, we argue that even with the entire $12 \times \mathrm{V} 1$ genes represented in the rnaseq1_MR network, the aggregate and non-aggregate microarray GCNs still outperform the latter considerably. Similarly, poorer performance in the RNA-seq GCNs may be due to smaller sample sizes (i.e. 300-700 samples) used in their construction as opposed to those used for microarrays
(1359 samples). However, we show that the microarray aggregate and correlation rank networks downsampled to that of corresponding RNA-seq network sizes were consistently better performing ((Fig. 2a, b, Supplementary Figure S5). It is noteworthy that relative underperformance of RNA-seq-derived networks as compared to microarrays in network reconstruction is not just observed here, but also in many other plant GCNs (e.g. Arabidopsis, maize, rice, and tomato) evaluated with other performance scoring strategies (Giorgi et al. 2013; Obayashi et al. 2018; Liesecke et al. 2019).

Although RNA-seq co-expression analysis is still relatively new and practical workflows for analysis remain poorly defined, we predict that grapevine RNA-seq GCNs still offer several advantages_-complementing microarrayderived GCN and revealing novel associations (Giorgi et al. 2013; Ballouz et al. 2015), but also circumvent potential cross-hybridization issues of microarray probe sets (Cramer et al. 2014). Indeed, despite the microarray aggregate outperforming the RNA-seq-derived networks both in the total number of terms and predictive performance, a large proportion of good-performing terms (AUROC $>0.7$ ) were in common between the two types of networks (Supplementary Figure S4). Nonetheless, there were still terms that were unique to and/or show noticeably stronger predictive performance (AUROC $>0.05$ ) in specific RNA-seq-derived networks (Supplementary Figure S4 and S8). These findings demonstrate the added benefit of RNA-seq GCNs in revealing associations that may be missed when using microarray networks as a sole source for gene function prediction. In practical terms, our recent endeavor to integrate new RNAseq to existing microarray GCNs also showcase that both are still relevant and functional, often playing complementary roles in enabling interesting biological and gene discoveries to be made (Wong et al. 2017, 2018; Sun et al. 2018, 2019; Vannozzi et al. 2018). However, a formal assessment of how recent recommendations or 'safe practices' for RNAseq GCN analysis (Giorgi et al. 2013; Ballouz et al. 2015; Huang et al. 2017) affect network performance in grapevine RNA-seq GCN is still needed.

Variants of GBA-based neighbor voting are now amongst the most popular network evaluation metrics (Ballouz et al. 2015, 2017; Verleyen et al. 2015; Obayashi et al. 2018), however, their use may not necessarily generalize broadly across different platforms and biological systems as a high quality functional annotation is often essential (Obayashi et al. 2018, 2019). Thus, the extensive use of GBA-based neighbor voting with MapMan BIN and Pfam throughout this study is perhaps the most easily critiqued. First, we opted for the use of MapMan BIN annotation (Thimm et al. 2004) over conventional ones (e.g. Gene Ontology, GO) for a few reasons as discussed previously (Thimm et al. 2004; Klie and Nikoloski 2012; Bolger et al. 2018). As such, we 
are not surprised to see a high-frequency of good BIN predictions (AUROC $>0.7$ ) across our evaluations, especially in the aggregate. As an alternative reference for performance assessment, we took advantage of Pfam and found comparable (albeit slightly lower) performance ratings with BIN annotation (Figs. 1, 2, and 3). Good congruence across highto low-performing terms with the rankings of their functionally-related BIN counterpart was also seen (Supplementary Table S1). Together, these findings suggest that the MapMan and Pfam annotations were already of sufficient quality and are equally suited for gene function prediction in grape. Nonetheless, we do not want to disregard other popular plant annotation schemes (e.g. GO and KEGG) that could also be potentially useful. Although exhaustively testing each annotation is beyond the scope of this study, we predict that performance outcomes using GBA-based neighbor voting are expected to be robust to most popular reference annotations (Bolger et al. 2018) with the well-annotated grapevine genome (Vitulo et al. 2014).

Similarly, the use of GBA-based neighbor voting as the sole performance metric is another element that is most easily critiqued. Therefore, using a functional enrichmentbased performance metric (Ulitsky and Shamir 2009) that is independent of GBA-based neighbor voting, we reassessed the BIN performance across individual, aggregated, and six microarray- and RNA-seq-derived networks. Remarkably, both enrichment-based F-measure and GBA-based AUROC metrics were highly congruent with one another across our extensive evaluations, often showing a strong linear relationship $\left(R^{2}\right.$ of 0.9-0.93) (Fig. 5). Our findings indicate that both metrics and underlying reference annotation are robust and equally suited for evaluating grapevine GCNs, however, the functional enrichment-based metric was orders of magnitude slower ( 10-20×, exact runtimes not shown) than the fully-vectorized implementation of GBA-based neighbor voting (Ballouz et al. 2017) used in this study. Therefore, the GBA-based AUROC metric is strongly favoured due to its exceptional scalability.

To demonstrate the functionality of the aggregated network, the function and transcriptional regulation of grapevine EXPANSIN genes were explored. Presently, the function of a few EXP genes (Suzuki et al. 2014) and their regulatory control have been elucidated (Nicolas et al. 2013). Large-scale transcriptional coordination of genes involved in the biosynthesis and regulation of primary and secondary cell wall can be found in many plants, often wherever cells are undergoing growth or modification of their cell wall composition (Mitchell et al. 2007; Ruprecht and Persson 2012; Cosgrove 2015; Sundell et al. 2017). Here, a strong co-expression relationship within the EXP family itself is highlighted between genes involved in various cell wall metabolic processes (e.g. degradation, modification, and pectin esterification), as well as those involved in transport and flavonoid/phenylpropanoid metabolism (Fig. 6). Across the EXP subnetworks, a wide range of TFs was also found to be co-expressed. However, a few (e.g. AP2/ERF, bZIP, bHLH, HB, NAC families) were frequently represented and have their corresponding CREs enriched in several EXP subnetworks.

It comes as no surprise as many grapevine EXP genes exhibit distinct expression patterns across various organs, development stages, and responses to the environment are potentially implicated in secondary cell wall formation, berry growth and ripening, response to the environment, among others (Dal Santo et al. 2013b). Coordinated regulation of other EXP co-expressed genes linked to these processes, i.e. modulation of major intrinsic proteins during berry growth and ripening (Schlosser et al. 2008; Nicolas et al. 2013) and flavonoid/phenylpropanoid metabolism during dehydration stress (Fasoli et al. 2012; Zenoni et al. 2016), provides robust evidence for a functional link between these groups of genes. While upstream control of many grapevine EXP genes remains to be ascertained, several members of prioritised TFs in other plants are known to regulate EXP pivotal for various facets of growth, development, and stress responses. For example, the regulation of EXP(s) during i. banana fruit ripening by an AP2/ERF TF, MaDREB2 (Kuang et al. 2017), ii. rose petal expansion and dehydration stress response by a NAC TF, RhNAC2 (Dai et al. 2012), and iii. growth and development in Arabidopsis by several bHLH TFs (Bai et al. 2012; Ikeda et al. 2012).

\section{Summary and future directions}

In summary, network aggregation methods offer unique opportunities for gene function prediction in grapevine. Across our extensive assessments, we found that the final aggregated network attained exceptional performance, outperforming networks constructed from all 33 individual experiments and a variety of state-of-the-art microarrayand RNA-seq-derived correlation rank (non-aggregate) networks. One major caveat of this study, albeit hard to avoid and commonly used, is that only one form of partitioning (i.e. experimental project-partitioned) and aggregation method (i.e. co-occurrence) were used. Nonetheless, other methods also exist (i.e. random- and k-means-based partitioning), and in some circumstances, networks constructed with them showed better performance than commonly used experimental project-partitioned co-occurrence aggregation methods (Liesecke et al. 2019). Thus, we predict that there may still be some room for improvement using such methods.

Another major caveat lies in the choice of using grapevine microarray datasets for this exercise because the latter platform will eventually be superseded by RNA-seq 
transcriptomes. Thus, defeating the primary purpose of network aggregation approaches (i.e. allowing emerging datasets to be efficiently incorporated). However, we argue that the microarray datasets not only provided a proof of concept to investigate the role of network aggregation but showed great promise for superior gene function inference using aggregated networks. We predict that the benefits of network aggregation methods will also be amendable to RNAseq transcriptomes, however, to what degree it may be more advantageous, will need to be ascertained in future studies.

To help guide future hypothesis-driven studies aimed at understanding the function and transcriptional regulatory networks of grapevine genes, we make our aggregate networks and associated metadata (e.g. functional BIN and CRE enrichment results) publicly available at https://sites .google.com/view/vtc-agg. We envisage that network aggregation will offer new and unique opportunities for superior gene function prediction in future grapevine functional genomics studies.

Acknowledgements The author would like to thank the anonymous reviewers for their helpful and constructive comments, José Tomás Matus for critically reviewing this work in its early stages, Marek Mutwil for the provision of the grape CoNekT dataset, and the grapevine research community for making various RNA-seq and microarray datasets publicly available.

Author contributions DCJW conceived the study, compiled and analysed the microarray data, performed the analysis, and drafted the manuscript.

\section{Compliance with ethical standards}

Conflict of interests The author declares that they have no conflict of interest.

Ethical approval This article does not contain any studies with human participants or animals performed by any of the authors.

\section{References}

Amato A, Cavallini E, Zenoni S, Finezzo L, Begheldo M, Ruperti B, Tornielli GB (2017) A grapevine TTG2-like WRKY transcription factor is involved in regulating vacuolar transport and flavonoid biosynthesis. Front Plant Sci 7:1979. https://doi.org/10.3389/ fpls.2016.01979

Aoki Y, Okamura Y, Tadaka S, Kinoshita K, Obayashi T (2016) ATTED-II in 2016: A plant coexpression database towards lineage-specific coexpression. Plant Cell Physiol 57:e5. https://doi. org/10.1093/pcp/pcv165

Bai M-Y, Fan M, Oh E, Wang Z-Y (2012) A triple helix-loop-helix/ basic helix-loop-helix cascade controls cell elongation downstream of multiple hormonal and environmental signaling pathways in Arabidopsis. Plant Cell 24:4917-4929. https://doi. org/10.1105/tpc.112.105163

Ballouz S, Verleyen W, Gillis J (2015) Guidance for RNA-seq coexpression network construction and analysis: Safety in numbers.
Bioinformatics 31:2123-2130. https://doi.org/10.1093/bioinforma tics/btv118

Ballouz S, Weber M, Pavlidis P, Gillis J (2017) EGAD: Ultra-fast functional analysis of gene networks. Bioinformatics 33:612-614. https://doi.org/10.1093/bioinformatics/btw695

Barrett T, Wilhite SE, Ledoux P, Evangelista C, Kim IF, Tomashevsky M, Marshall KA, Phillippy KH, Sherman PM, Holko M, Yefanov A, Lee H, Zhang N, Robertson CL, Serova N, Davis S, Soboleva A (2013) NCBI GEO: archive for functional genomics data sets-update. Nucleic Acids Res 41:D991-D995. https://doi. org/10.1093/nar/gks1193

Bolger ME, Arsova B, Usadel B (2018) Plant genome and transcriptome annotations: from misconceptions to simple solutions. Brief Bioinform 19:437-449. https://doi.org/10.1093/bib/bbw135

Carvalho BS, Irizarry RA (2010) A framework for oligonucleotide microarray preprocessing. Bioinformatics 26:2363-2367. https:// doi.org/10.1093/bioinformatics/btq431

Cosgrove DJ (2015) Plant expansins: diversity and interactions with plant cell walls. Curr Opin Plant Biol 25:162-172. https://doi. org/10.1016/j.pbi.2015.05.014

Cramer GR, Ghan R, Schlauch KA, Tillett RL, Heymann H, Ferrarini A, Delledonne M, Zenoni S, Fasoli M, Pezzotti M (2014) Transcriptomic analysis of the late stages of grapevine (Vitis vinifera cv. Cabernet Sauvignon) berry ripening reveals significant induction of ethylene signaling and flavor pathways in the skin. BMC Plant Biol 14:1-21. https://doi.org/10.1186/s12870-014-0370-8

Dai F, Zhang C, Jiang X, Kang M, Yin X, Lu P, Zhang X, Zheng Y, Gao J (2012) RhNAC2 and RhEXPA4 are involved in the regulation of dehydration tolerance during the expansion of rose petals. Plant Physiol 160:2064-2082. https://doi.org/10.1104/ pp.112.207720

Dal Santo S, Palliotti A, Zenoni S, Tornielli GB, Fasoli M, Paci P, Tombesi S, Frioni T, Silvestroni O, Bellincontro A, d'Onofrio C, Matarese F, Gatti M, Poni S, Pezzotti M (2016) Distinct transcriptome responses to water limitation in isohydric and anisohydric grapevine cultivars. BMC Genomics 17:1-19. https://doi. org/10.1186/s12864-016-3136-X

Dal Santo S, Tornielli GB, Zenoni S, Fasoli M, Farina L, Anesi A, Guzzo F, Delledonne M, Pezzotti M (2013a) The plasticity of the grapevine berry transcriptome. Genome Biol 14:r54. https://doi. org/10.1186/gb-2013-14-6-r54

Dal Santo S, Vannozzi A, Tornielli GB, Fasoli M, Venturini L, Pezzotti M, Zenoni S (2013b) Genome-wide analysis of the expansin gene superfamily reveals grapevine-specific structural and functional characteristics. PLoS ONE 8:e62206. https://doi.org/10.1371/ journal.pone.0062206

Fasoli M, Dal Santo S, Zenoni S, Tornielli GB, Farina L, Zamboni A, Porceddu A, Venturini L, Bicego M, Murino V, Ferrarini A, Delledonne M, Pezzotti M (2012) The grapevine expression atlas reveals a deep transcriptome shift driving the entire plant into a maturation program. Plant Cell 24:3489-3505. https://doi. org/10.1105/tpc. 112.100230

Gibson SM, Ficklin SP, Isaacson S, Luo F, Feltus FA, Smith MC (2013) Massive-scale gene co-expression network construction and robustness testing using random matrix theory. PLoS ONE. https://doi.org/10.1371/journal.pone.0055871

Gillis J, Pavlidis P (2012) "Guilt by association" is the exception rather than the rule in gene networks. PLoS Comput Biol 8:e1002444. https://doi.org/10.1371/journal.pcbi.1002444

Gillis J, Pavlidis P (2011) The role of indirect connections in gene networks in predicting function. Bioinformatics 27:1860-1866. https://doi.org/10.1093/bioinformatics/btr288

Giorgi FM, Del Fabbro C, Licausi F (2013) Comparative study of RNA-seq- and Microarray-derived coexpression networks in Arabidopsis thaliana. Bioinformatics 29:717-724. https://doi. org/10.1093/bioinformatics/btt053 
Grimplet J, Martínez-zapater JM, Carmona MJ (2016) Structural and functional annotation of the MADS-box transcription factor family in grapevine. BMC Genomics 17:80. https://doi. org/10.1186/s12864-016-2398-7

Huang J, Vendramin Alegre S, Shi L, McGinnis K (2017) Construction and optimization of large gene co-expression network in maize using RNA-Seq data. Plant Physiol 175:00825.2017. https://doi.org/10.1104/pp.17.00825

Ikeda M, Fujiwara S, Mitsuda N, Ohme-Takagi M (2012) A Triantagonistic basic helix-loop-helix system regulates cell elongation in Arabidopsis. Plant Cell 24:4483-4497. https://doi.org/10.1105/ tpc. 112.105023

Klie S, Nikoloski Z (2012) The choice between MapMan and Gene ontology for automated gene function prediction in plant science. Front Genet 3:1-14. https://doi.org/10.3389/fgene .2012 .00115

Kuang JF, Chen JY, Liu XC, Han YC, Xiao YY, Shan W, Tang Y, Wu KQ, He JX, Lu WJ (2017) The transcriptional regulatory network mediated by banana (Musa acuminata) dehydration-responsive element binding (MaDREB) transcription factors in fruit ripening. New Phytol 214:762-781. https://doi.org/10.1111/nph.14389

Liesecke F, De Craene JO, Besseau S, Courdavault V, Clastre M, Vergès V, Papon N, Giglioli-Guivarc'h N, Glévarec G, Pichon O, Dugé de Bernonville T (2019) Improved gene co-expression network quality through expression dataset down-sampling and network aggregation. Sci Rep 9:1-16. https://doi.org/10.1038/ s41598-019-50885-8

Lohse M, Nagel A, Herter T, May P, Schroda M, Zrenner R, Tohge T, Fernie AR, Stitt M, Usadel B (2014) Mercator: a fast and simple web server for genome scale functional annotation of plant sequence data. Plant Cell Environ 37:1250-1258. https://doi. org/10.1111/pce.12231

Loyola R, Herrera D, Mas A, Wong DCJ, Höll J, Cavallini E, Amato A, Azuma A, Ziegler T, Aquea F, Castellarin SD, Bogs J, Tornielli GB, Peña-Neira A, Czemmel S, Alcalde JA, Matus JT, Arce-Johnson P (2016) The photomorphogenic factors UV-B RECEPTOR 1, ELONGATED HYPOCOTYL 5, and HY5 HOMOLOGUE are part of the UV-B signalling pathway in grapevine and mediate flavonol accumulation in response to the environment. J Exp Bot. https://doi.org/10.1093/jxb/erw307

Malacarne G, Coller E, Czemmel S, Vrhovsek U, Engelen K, Goremykin V, Bogs J, Moser C (2016) The grapevine VvibZIPC22 transcription factor is involved in the regulation of flavonoid biosynthesis. J Exp Bot 67:3509-3522. https://doi.org/10.1093/jxb/ erw 181

Malacarne G, Pilati S, Valentini S, Asnicar F, Moretto M, Sonego P, Masera L, Cavecchia V, Blanzieri E, Moser CM (2018) Discovering causal relationships in grapevine expression data to expand gene networks. A case study: four networks related to climate change. Front Plant Sci 9:1385. https://doi.org/10.3389/ FPLS.2018.01385

Massonnet M, Fasoli M, Tornielli GB, Altieri M, Sandri M, Zuccolotto P, Paci P, Gardiman M, Zenoni S, Pezzotti M (2017) Ripening transcriptomic program in red and white grapevine varieties correlates with berry skin anthocyanin accumulation. Plant Physiol 174:2376-2396. https://doi.org/10.1104/pp.17.00311

Mitchell RAC, Dupree P, Shewry PR (2007) A novel bioinformatics approach identifies candidate genes for the synthesis and feruloylation of arabinoxylan. Plant Physiol 144:43-53. https://doi. org/10.1104/pp.106.094995

Moretto M, Sonego P, Pilati S, Malacarne G, Costantini L, Grzeskowiak L, Bagagli G, Grando MS, Moser C, Engelen K (2016) VESPUCCI: Exploring patterns of gene expression in grapevine. Front Plant Sci 7:633. https://doi.org/10.3389/fpls.2016.00633

Nicolas P, Lecourieux D, Gomès E, Delrot S, Lecourieux F (2013) The grape berry-specific basic helix-loop-helix transcription factor
VvCEB1 affects cell size. J Exp Bot 64:991-1003. https://doi. org/10.1093/jxb/ers374

O’Malley RC, Huang SC, Song L, Lewsey MG, Bartlett A, Nery JR, Galli M, Gallavotti A, Ecker JR (2016) Cistrome and epicistrome features shape the regulatory DNA landscape. Cell 165:12801292. https://doi.org/10.1016/j.cell.2016.04.038

Obayashi T, Aoki Y, Tadaka S, Kagaya Y, Kinoshita K (2018) ATTEDII in 2018: a plant coexpression database based on investigation of the statistical property of the Mutual Rank Index. Plant Cell Physiol 59:e3. https://doi.org/10.1093/pcp/pcx191

Obayashi T, Kagaya Y, Aoki Y, Tadaka S, Kinoshita K (2019) COXPRESdb v7: A gene coexpression database for 11 animal species supported by 23 coexpression platforms for technical evaluation and evolutionary inference. Nucleic Acids Res 47:D55-D62. https ://doi.org/10.1093/nar/gky1155

Obayashi T, Kinoshita K (2009) Rank of correlation coefficient as a comparable measure for biological significance of gene coexpression. DNA Res 16:249-260. https://doi.org/10.1093/dnares/dsp01 6

Ohyanagi H, Takano T, Terashima S, Kobayashi M, Kanno M, Morimoto K, Kanegae H, Sasaki Y, Saito M, Asano S, Ozaki S, Kudo T, Yokoyama K, Aya K, Suwabe K, Suzuki G, Aoki K, Kubo Y, Watanabe M, Matsuoka M, Yano K (2015) Plant omics data center: an integrated web repository for interspecies gene expression networks with NLP-based curation. Plant Cell Physiol 56:e9. https://doi.org/10.1093/pcp/pcu188

Palumbo MC, Zenoni S, Fasoli M, Massonnet M, Farina L, Castiglione F, Pezzotti M, Paci P (2014) Integrated network analysis identifies fight-club nodes as a class of hubs encompassing key putative switch genes that induce major transcriptome reprogramming during grapevine development. Plant Cell Online 26:4617-4635. https://doi.org/10.1105/tpc.114.133710

Proost S, Mutwil M (2018) CoNekT: An open-source framework for comparative genomic and transcriptomic network analyses. Nucleic Acids Res 46:W133-W140. https://doi.org/10.1093/nar/ gky336

Ruprecht C, Persson S (2012) Co-expression of cell-wall related genes: new tools and insights. Front Plant Sci 3:1-7. https://doi. org/10.3389/fpls.2012.00083

Ruprecht C, Proost S, Hernandez-Coronado M, Ortiz-Ramirez C, Lang D, Rensing SA, Becker JD, Vandepoele K, Mutwil M (2017) Phylogenomic analysis of gene co-expression networks reveals the evolution of functional modules. Plant J 90:447-465. https://doi. org/10.1111/tpj.13502

Rustici G, Kolesnikov N, Brandizi M, Burdett T, Dylag M, Emam I, Farne A, Hastings E, Ison J, Keays M, Kurbatova N, Malone J, Mani R, Mupo A, PedroPereira R, Pilicheva E, Rung J, Sharma A, Tang YA, Ternent T, Tikhonov A, Welter D, Williams E, Brazma A, Parkinson H, Sarkans U (2013) ArrayExpress update-trends in database growth and links to data analysis tools. Nucleic Acids Res 41:D987-D990. https://doi.org/10.1093/nar/gks1174

Savoi S, Wong DCJ, Arapitsas P, Miculan M, Bucchetti B, Peterlunger E, Fait A, Mattivi F, Castellarin SD (2016) Transcriptome and metabolite profiling reveals that prolonged drought modulates the phenylpropanoid and terpenoid pathway in white grapes (Vitis vinifera L.). BMC Plant Biol 16:67. https://doi.org/10.1186/s1287 0-016-0760-1

Savoi S, Wong DCJ, Degu A, Herrera JC, Bucchetti B, Peterlunger E, Fait A, Mattivi F, Castellarin SD (2017) Multi-omics and integrated network analyses reveal new insights into the systems relationships between metabolites, structural genes, and transcriptional regulators in developing grape berries (Vitis vinifera L.) exposed to water deficit. Front Plant Sci 8:1124. https://doi. org/10.3389/fpls.2017.01124

Schlosser J, Olsson N, Weis M, Reid K, Peng F, Lund S, Bowen P (2008) Cellular expansion and gene expression in the developing 
grape (Vitis vinifera L.). Protoplasma 232:255-265. https://doi. org/10.1007/s00709-008-0280-9

Serin EAR, Nijveen H, Hilhorst HWM, Ligterink W (2016) Learning from co-expression networks: possibilities and challenges. Front Plant Sci 7:1-18. https://doi.org/10.3389/fpls.2016.00444

Sun X, Matus JT, Wong DCJ, Wang Z, Chai F, Zhang L, Fang T, Zhao L, Wang Y, Han Y, Wang Q, Li S, Liang Z, Xin H (2018) The GARP/MYB-related grape transcription factor AQUILO improves cold tolerance and promotes the accumulation of raffinose family oligosaccharides. J Exp Bot 69:1749-1764. https:// doi.org/10.1093/jxb/ery020

Sun X, Zhang L, Wong DCJ, Wang Y, Zhu Z, Xu G, Wang Q, Li S, Liang Z, Xin H (2019) The ethylene response factor VaERF092 from Amur grape regulates the transcription factor VaWRKY33, improving cold tolerance. Plant J. https://doi.org/10.1111/ tpj. 14378

Sundell D, Street NR, Kumar M, Mellerowicz EJ, Kucukoglu M, Johnsson C, Kumar V, Mannapperuma C, Delhomme N, Nilsson O, Tuominen H, Pesquet E, Fischer U, Niittylä T, Sundberg B, Hvidsten TR (2017) AspWood: high-spatial-resolution transcriptome profiles reveal uncharacterized modularity of wood formation in Populus tremula. Plant Cell 29:1585-1604. https://doi. org/10.1105/tpc. 17.00153

Suzuki H, Oshita E, Fujimori N, Nakajima Y, Kawagoe Y, Suzuki S (2014) Grape expansins, VvEXPA14 and VvEXPA18 promote cell expansion in transgenic Arabidopsis plant. Plant Cell Tissue Organ Cult 120:1077-1085. https://doi.org/10.1007/s1124 $0-014-0662-6$

Thimm O, Bläsing O, Gibon Y, Nagel A, Meyer S, Krüger P, Selbig J, Müller LA, Rhee SY, Stitt M (2004) MAPMAN: a user-driven tool to display genomics data sets onto diagrams of metabolic pathways and other biological processes. Plant J 37:914-939. https://doi.org/10.1111/j.1365-313X.2004.02016.x

Ulitsky I, Shamir R (2009) Identifying functional modules using expression profiles and confidence-scored protein interactions. Bioinformatics 25:1158-1164. https://doi.org/10.1093/bioinforma tics/btp118

Usadel B, Obayashi T, Mutwil M, Giorgi FM, Bassel GW, Tanimoto M, Chow A, Steinhauser D, Persson S, Provart NJ (2009) Coexpression tools for plant biology: opportunities for hypothesis generation and caveats. Plant Cell Environ 32:1633-1651. https ://doi.org/10.1111/j.1365-3040.2009.02040.x

Vannozzi A, Wong DCJ, Holl J, Hmmam I, Matus JT, Bogs J, Ziegler T, Dry I, Barcaccia G, Lucchin M (2018) Combinatorial regulation of stilbene synthase genes by WRKY and MYB transcription factors in grapevine (Vitis vinifera L.). Plant Cell Physiol 59:1043-1059. https://doi.org/10.1093/pcp/pcy045

Verleyen W, Ballouz S, Gillis J (2015) Measuring the wisdom of the crowds in network-based gene function inference. Bioinformatics 31:745-752. https://doi.org/10.1093/bioinformatics/btu715

Vitulo N, Forcato C, Carpinelli EC, Telatin A, Campagna D, D'Angelo M, Zimbello R, Corso M, Vannozzi A, Bonghi C, Lucchin M, Valle G (2014) A deep survey of alternative splicing in grape reveals changes in the splicing machinery related to tissue, stress condition and genotype. BMC Plant Biol 14:99. https://doi. org/10.1186/1471-2229-14-99

Wong DCJ, Lopez Gutierrez R, Gambetta GA, Castellarin SD (2017) Genome-wide analysis of cis-regulatory element structure and discovery of motif-driven gene co-expression networks in grapevine. DNA Res 24:311-326. https://doi.org/10.1093/dnares/dsw061

Wong DCJ, Matus JT (2017) Constructing integrated networks for identifying new secondary metabolic pathway regulators in grapevine : Recent applications and future opportunities. Front Plant Sci 8:505. https://doi.org/10.3389/fpls.2017.00505

Wong DCJ, Schlechter R, Vannozzi A, Höll J, Hmmam I, Bogs J, Tornielli GB, Castellarin SD, Matus JT (2016) A systems-oriented analysis of the grapevine R2R3-MYB transcription factor family uncovers new insights into the regulation of stilbene accumulation. DNA Res 23:451-466. https://doi.org/10.1093/dnares/dsw02 8

Wong DCJ, Sweetman C, Drew DP, Ford CM (2013) VTCdb: a gene co-expression database for the crop species Vitis vinifera (grapevine). BMC Genomics 14:882. https://doi. org/10.1186/1471-2164-14-882

Wong DCJ, Zhang L, Merlin I, Castellarin SD, Gambetta GA (2018) Structure and transcriptional regulation of the major intrinsic protein gene family in grapevine. BMC Genomics 19:248. https://doi. org/10.1186/s12864-018-4638-5

Zenoni S, Fasoli M, Guzzo F, Dal Santo S, Amato A, Anesi A, Commisso M, Herderich M, Ceoldo S, Avesani L, Pezzotti M, Tornielli GB (2016) Disclosing the molecular basis of the postharvest life of berry in different grapevine genotypes. Plant Physiol 172:18211843. https://doi.org/10.1104/pp.16.00865

Publisher's Note Springer Nature remains neutral with regard to jurisdictional claims in published maps and institutional affiliations. 